\title{
Thermal resummation and phase transitions
}

\author{
David Curtin $^{1,2, a}$, Patrick Meade ${ }^{3, b}$, Harikrishnan Ramani ${ }^{3,4,5, c} \mathbb{C}_{\mathbb{C}}$ \\ ${ }^{1}$ Maryland Center for Fundamental Physics, University of Maryland, College Park, MD 20742, USA \\ ${ }^{2}$ Department of Physics, University of Toronto, Toronto, ON M5S 1A7, Canada \\ ${ }^{3}$ C. N. Yang Institute for Theoretical Physics, SUNY Stony Brook, Stony Brook, NY 11794, USA \\ ${ }^{4}$ Department of Physics, Berkeley Center for Theoretical Physics, University of California, Berkeley, CA 94720, USA \\ 5 Theoretical Physics Group, Lawrence Berkeley National Laboratory, Berkeley, CA 94720, USA
}

Received: 6 August 2018 / Accepted: 19 September 2018 / Published online: 27 September 2018

(C) The Author(s) 2018

\begin{abstract}
The consequences of phase transitions in the early universe are becoming testable in a variety of manners, from colliders physics to gravitational wave astronomy. In particular one phase transition we know of, the electroweak phase transition (EWPT), could potentially be first order in BSM scenarios and testable in the near future. If confirmed this could provide a mechanism for baryogenesis, which is one of the most important outstanding questions in physics. To reliably make predictions it is necessary to have full control of the finite temperature scalar potentials. However, as we show the standard methods used in BSM physics to improve phase transition calculations, resumming hard thermal loops, introduces significant errors into the scalar potential. In addition, the standard methods make it impossible to match theories to an EFT description reliably. In this paper we define a thermal resummation procedure based on partial dressing (PD) for general BSM calculations of phase transitions beyond the high-temperature approximation. Additionally, we introduce the modified optimized partial dressing (OPD) procedure, which is numerically nearly as efficient as old incorrect methods, while yielding identical results to the full PD calculation. This can be easily applied to future BSM studies of phase transitions in the early universe. As an example, we show that in unmixed singlet scalar extensions of the SM, the (O)PD calculations make new phenomenological predictions compared to previous analyses. An important future application is the study of EFTs at finite temperature.
\end{abstract}

\section{Contents}

1 Introduction . . . . . . . . . . . 1

2 Review: calculating the electroweak phase transition 4

\footnotetext{
a e-mail: dcurtin@physics.utoronto.ca

b e-mail: patrick.meade@stonybrook.edu

c e-mail: harikrishnan.ramani@stonybrook.edu
}

2.1 Tree-level potential . . . . . . . . . . . . . . . 4

2.2 Coleman Weinberg potential . . . . . . . . 4

2.3 Finite temperature . . . . . . . . . . . . . 5

2.4 Resummation of the thermal mass: truncated full dressing (TFD) . . . . . . . . . . . 6

2.5 Types of electroweak phase transitions . . . . . 7

2.6 Problems with the standard one-loop TFD calculation of the phase transition . . . . . . . 9 9

3 Formal aspects of finite-temperature mass resummation . . . . . . . . . . . . 10

3.1 Tadpole resummation in $\phi^{4}$ theories $\ldots \ldots .11$ 3.1.1 Partial dressing results for $N=1 \ldots 11$

3.1.2 Comparing resummation schemes . . . 12

3.2 A general resummation procedure for BSM theories . . . . . . . . . . . 13

3.3 Future directions . . . . . . . . . . . . 15

3.4 Comparison with dimensional reduction . . . . 15

4 Computing the strength of the phase transition . . 16

4.1 Zero-temperature calculation . . . . . . . . 16

4.2 Finite-temperature calculation . . . . . . . . 17

4.2.1 Truncated full dressing (TFD) . . . . . . 17

4.2.2 Partial dressing (PD) . . . . . . . . 18

4.2.3 Optimized partial dressing (OPD) $\ldots . .18$

4.3 Comparing resummation schemes . . . . . 2 20

5 Physical consequences . . . . . . . . . . . 2 21

6 Conclusions . . . . . . . . . . . . . . 24

Appendix A: Instruction manual for optimized partial dressing calculation of phase transition . . . . . 26

References ............... 27

\section{Introduction}

Thermal phase transitions are ubiquitous phenomena in nature, but in fundamental physics they are difficult to study, and very few are known. In the SM there are phase transitions associated with QCD and the EW symmetry. The QCD 
phase transition can be studied directly in heavy ion collisions, with rapid progress over the last decade [1]. However, the EW phase transition (EWPT) is far out of reach of direct testability. Going beyond the Standard Model (BSM), the nature of the EW phase transition could change, and there could be additional phase transitions unrelated to the EWPT but still screened from us by the CMB. Nevertheless, even without direct measurements of the EWPT in the near future these phenomena can be indirectly studied, with profound consequences for our understanding of the early universe. The EWPT and other phase transitions can have correlated signals detectable at current and future colliders, and in the burgeoning field of gravitational wave astronomy.

Therefore, it is important to have as much control of the underlying finite-temperature quantum field theory (FTQFT) calculations as possible, so that potential signals are reliably understood and predicted. This is the aim of this paper, and we will introduce new methods in FTQFT to capture the effects of BSM physics on phase transitions. While our results will be general, we single out the EWPT for special study given its possible deep connection to another fundamental question in particle physics.

One of the most profound mysteries in particle physics is our mere existence, and that of all baryons in the universe. A dynamical explanation for our universe containing an excess of matter over antimatter requires BSM physics. At some time in the history of the primordial plasma, after reheating but before Big Bang Nucleosynthesis ( $T \sim \mathrm{GeV})$, a mechanism of baryogenesis has to create the observed baryon asymmetry [2,3] of

$\eta=\frac{n_{B}-n \bar{B}}{n_{\gamma}} \sim 10^{-9}$.

This requires the three Sakharov conditions $[4,5]$ to be satisfied: baryon number $(B)$ violation, $C P$ violation, and a sufficiently sharp departure from thermal equilibrium.

Electroweak Baryogenesis [6-13] is a very appealing possibility, since all involved processes must occur near the weak scale, making it in principle testable. (See [14-19] for reviews.) In the SM, high temperature effects stabilize the Higgs field at the origin, restoring electroweak symmetry $[20,21]$. In this high-temperature unbroken phase, the SM in fact contains a $(B+L)$-violating process in the form of nonperturbative sphaleron transitions, which can convert a chiral asymmetry into a baryon asymmetry. The EWPT from the unbroken to the broken phase at $T \sim 100 \mathrm{GeV}$ provides, in principle, a departure from thermal equilibrium. In the presence of sufficient $C P$-violation in the plasma, a baryon excess can be generated.

EWBG cannot function within the SM alone. There is insufficient $C P$-violation (see for example [16]), and the EWPT is not first order for $m_{h} \gtrsim 70 \mathrm{GeV}$ [22,23]. Additional BSM physics is required to generate a strong phase transition (PT) and supply additional $C P$-violating interactions in the plasma.

Many theories have been proposed to fulfill these requirements of EWBG, including extensions of the scalar sector with additional singlets [24-37] (which can be embedded in supersymmetric models [38-44]), two-Higgs doublet models $[45,46]$, triplet extensions [47-49], and the well-known lightstop scenario in the MSSM [50-60] which is now excluded [61-64]. To determine whether a particular, complete model can successfully account for the observed baryon asymmetry, the temperature-dependent Higgs potential and the resulting nature of the phase transition have to be carefully calculated to determine the sphaleron energy as well as the bubble nucleation rate and profile. This information serves as an input to solve a set of plasma transport equations, which determine the generated baryon asymmetry of the universe (BAU). The full calculation is very intricate, with many unresolved theoretical challenges (see e.g. [55-57,65-79]).

The sectors of a theory which generate the strong phase transition, and generate baryon number via $C P$-violating interactions in the plasma, do not have to be connected (though they can be). Since one of the most appealing features of EWBG is its testability, it makes sense to consider these two conditions and their signatures separately. The ultimate aim is a model-independent understanding of the collider, low-energy, and cosmological signatures predicted by all the various incarnations of EWBG.

We will focus on the strong electroweak phase transition. If it is first order, there is a critical temperature $T_{c}$ where the Higgs potential has two degenerate minima $h=0$ and $v_{c}$, separated by an energy barrier. As the temperature decreases, the minimum away from the origin becomes the true vacuum, the Higgs field tunnels to the broken phase, and bubbles of true vacuum expand to fill the universe. A necessary condition for avoiding baryon washout is that $v_{c}$ is sufficiently large to suppress sphalerons. Specifically, a BSM theory which realizes EWBG has to satisfy

$\frac{v_{c}}{T_{c}}>0.6-1.6$

In most cases we will adopt the lower value of 0.6 as our cutoff [80] to be as inclusive as possible (though it is sometimes instructive to examine the parameter space that survives the more standard $v_{c} / T_{c}>1$ criterion.) This is a useful way of checking whether a given BSM scenario is a viable candidate for EWBG, as well as determining the correlated signatures we could measure today.

Computing this ratio seems like a straightforward exercise, and a standard recipe has been adopted in the literature for computing the EWPT in BSM models (see e.g. [18] for a review). This involves constructing the one-loop effective Higgs potential at finite temperature by using a well-known generalization of the standard Coleman-Weinberg potential; 
possibly including a selection of the most important higherloop effects and/or RG-improvements; and resumming an important set of contributions called hard thermal loops.

We carefully review this calculation in Sect. 2. Our focus is the resummation of hard thermal loops. The standard procedure, which we call truncated full dressing (TFD), involves a very simple computation of thermal masses $\Pi_{i} \sim T^{2}$ for particles $i$ in the plasma, to leading order in the high-temperature approximation, and inserting them back into the effective potential [81-83].

Various extensions of this simple recipe, to include higherorder corrections in temperature or coupling, have been explored roughly twenty years ago in the context of $\phi^{4}$ theories [84-89]. However, possibly because the consensus on the (most) correct generalization seemed unclear, and the involved calculations seemed onerous to perform for every BSM theory, these improvements have not found wide application in the study of the EWPT in general BSM scenarios.

We revisit these issues in a modern light, with a focus on the study of general BSM effects which can induce a strong EWPT. A simple and easily implementable extension of the TFD calculation is urgently required for two reasons: to correctly determine the phenomenology of EWBG, and to understand effective field theory (EFT) at finite temperature.

Since the high-temperature expansion of $\Pi_{i}$ is truncated at the leading term, it is only accurate to $\mathcal{O}(m / T)$. This can easily be $\sim 40 \%$ at $T=T_{c}$, and vary with the Higgs field since its VEV determines particle masses. While this does not directly translate to a corresponding error on the full effective potential, an important class of (particularly testable) EWBG theories generates a strong EWPT via a partial cancellation between $\Pi_{i}$ and tree-level parameters. In this case, accurate determination of the thermal masses, and their $h$ dependence, is clearly necessary to have confidence in the results of the phase transition computation, and hence the observables correlated with EWBG.

Effective field theories (EFTs) are a powerful tool to parameterize general new physics effects at zero temperature as a set of non-renormalizable operators involving SM fields. To understand the signatures of EWBG in a modelindependent fashion, one would like to extend such an EFT analysis to finite temperature. Early attempts like [90-93] suggested that a $|H|^{6}$ operator could induce a strong EWPT in correlation with sizable deviations in the cubic Higgs selfcoupling, which could be detected with the next generation of future lepton [94,95] and $100 \mathrm{TeV}$ [96-98] colliders, or even the HL-LHC [99-104]. Unfortunately, EFTs at finite temperature are very poorly understood. For example, the effects of a particle with a mass of $300 \mathrm{GeV}$ are quite well described in an EFT framework for collider experiments with $\sqrt{s}=100 \mathrm{GeV}$, but it seems doubtful that this is the case for temperatures of $T=100 \mathrm{GeV}$, since thermal fluctuations can excite modes somewhat heavier than $T$. Without understanding these effects in detail, we cannot know the EFT's radius of convergence in field space or temperature, and hence know whether its predictions regarding the EWPT can be trusted. The authors of [105] investigated the validity of finite temperature EFT by comparing it to a singlet scalar model in the TFD prescription. Since the assumptions of the high-temperature approximation $(T \gg m)$ for $\Pi_{i}$ in TFD are fundamentally incompatible with the assumptions of an finite-temperature EFT analysis $(T \ll m)$, careful study of these decoupling effects during a phase transition, and EFT matching at finite temperature, requires a more complete treatment of thermal masses.

In this work, we develop a consistent, easily implementable procedure for the numerical computation and resummation of thermal masses in general BSM theories, beyond leading order in temperature and coupling.

We examine two competing approaches which were proposed in the context of $\phi^{4}$ theories: full dressing (FD) [8486] and partial dressing (PD) [87]. We verified the claims of [87] that PD avoids the problem of miscounting diagrams beyond one-loop order [88,89], and that it generalizes beyond $\mathcal{O}\left(T^{2}\right)$. We therefore focus on PD. We review its formal underpinnings in Sect. 3 and outline how to generalize it to BSM theories, in general without relying on the high-temperature approximation. Additionally, in Sect. 3.4, we discuss the comparisons of this method to dimensional reduction which is often employed to investigate the high $T$ limit [23,106-110].

Applying the PD procedure beyond the high-temperature approximation requires numerically solving a type of finitetemperature gap equation. We outline the implementation of this calculation in Sect. 4 in the context of a specific BSM benchmark model. Computing the strength of the EWPT with PD is extremely numerically intensive, necessitating the use of a custom-built $\mathrm{C}++$ code. This allows us to study the importance of resummed finite-temperature effects for the phase transition, but is impractical for future BSM studies. We show it is possible to modify PD by extending the gap equation and implementing certain approximations. This greatly increases numerical reliability, while reducing CPU cost by several orders of magnitude. We call this updated resummation procedure optimized partial dressing (OPD) and show it is equivalent to PD for BSM studies of the EWPT.

OPD is only slightly more CPU-intensive than the standard TFD calculation used in most studies of the EWPT to date, and very easy to implement in Mathematica. We hope that this calculation, which is explained in Sect. 4 and summarized in the form of an instruction manual in Appendix A, will be useful in the future study of the EWPT for BSM theories.

The BSM model we use to develop and evaluate the PD and OPD resummation schemes is the SM with $N_{S}$ added singlets transforming under an unbroken $O\left(N_{S}\right)$ symmetry 
in our vacuum (or $\mathbb{Z}_{2}$ if $N_{S}=1$ ) and coupling to the SM via a quartic Higgs portal $\lambda_{H S}|H|^{2} S_{i}^{2}$ without Higgs mixing. This benchmark model serves as a useful "worst-case scenario" for the collider phenomenology of EWBG, since it can produce a strong EWPT in a variety of ways which are representative of more complete theories, while generating the minimal set of collider signatures consistent with EWBG. The authors of [111] studied this scenario with $N_{S}=1$ using the TFD calculation, making progress towards a "phenomenological no-lose theorem for EWBG" by showing that the future $100 \mathrm{TeV}$ and lepton colliders could probe its EWBGcompatible parameter space completely. We update and generalize this phenomenological analysis for $N_{S} \geq 1$ in the PD scheme. As shown in Sect. 5, the "no-lose theorem" is strengthened, with EWPTs caused by larger numbers of scalars being easier to detect at colliders.

Phenomenologically, the main lessons of the updated PD calculation are that the detailed correlations between a strong EWPT and collider observables can be significantly shifted, especially in more complete theories of EWBG than our SM $+N_{S} \times S$ benchmark model. Furthermore, two-step transitions are more prevalent than suggested by earlier TFD calculations. This raises the exciting prospect of discovering the traces of a strong two-step transition with gravitational wave observations [112,113]. Finally, unlike (O)PD, the TFD calculation overestimates the reliability of the finite-temperature EWPT calculation, underlining the importance of tracking error terms when computing the strength of the PT.

This paper is structured as follows. The standard TFD calculation of the EWPT is pedagogically reviewed in Sect. 2. Sect. 3 lays the formal groundwork of the PD scheme, while the implementation of the full calculation and its extension to the OPD scheme is described in Sect. 4. The differences in physical predictions between the standard TFD and the new (O)PD calculation are explored in Sect. 5. We conclude in Sect. 6, and provide an instruction manual for easy implementation of the OPD calculation for the EWPT in Appendix A.

\section{Review: calculating the electroweak phase transition}

We now review the standard computation of the finitetemperature Higgs potential in BSM theories (see e.g. [18]). We call the leading-order thermal mass resummation [81-83] TFD, to contrast with the PD procedure which we review and develop further in Sects. 3 and 4. This will make plain some important shortcomings of TFD.

As a BSM benchmark, we consider the SM with $N_{S}$ added real SM-singlet scalar fields $S_{i}$ obeying an $O\left(N_{S}\right)$ symmetry (or $\mathbb{Z}_{2}$ if $N_{S}=1$ ). We are also interested in regions of parameter space where this symmetry is unbroken in the zerotemperature vacuum of our universe today (i.e. $\left\langle S_{i}\right\rangle=0$ ).
This forbids Higgs-Singlet mixing, which significantly simplifies several formal aspects of thermal mass resummation. Unmixed singlet extensions also represent a useful "phenomenological nightmare scenario" for EWBG [111] with minimal experimental signatures. We show in Sect. 5 that this model can nonetheless be completely probed by the next generation of colliders.

\subsection{Tree-level potential}

The tree-level scalar potential is

$$
\begin{aligned}
V_{0}= & -\mu^{2}|H|^{2}+\lambda|H|^{4}+\frac{1}{2} \mu_{S}^{2}\left(S_{i} S_{i}\right) \\
& +\frac{1}{4} \lambda_{S}\left(S_{i} S_{i}\right)^{2}+\lambda_{H S}|H|^{2}\left(S_{i} S_{i}\right) .
\end{aligned}
$$

We focus on the real $h$ component of the SM Higgs doublet $H=\left(G^{+},\left(h+i G^{0}\right) / \sqrt{2}\right)$ which acquires a VEV during EWSB. Without loss of generality, we also assume that any excursion in S-field-space occurs along the $S_{0}$ direction. Therefore, the relevant part of the tree-level potential is

$V_{0}=-\frac{1}{2} \mu^{2} h^{2}+\frac{1}{4} \lambda h^{4}+\frac{1}{2} \mu_{S}^{2} S_{0}^{2}+\frac{1}{4} \lambda_{S} S_{0}^{4}+\frac{1}{2} \lambda_{H S} h^{2} S_{0}^{2}$.

(Of course, Eq. (2.1) determines the form of the scalar masses $m_{G^{+}}^{2}\left(h, S_{0}\right), m_{S_{i}}^{2}\left(h, S_{0}\right), \ldots$ which determine the form of one-loop contributions as outlined below.) Our aim is to obtain the effective potential $V_{\text {eff }}(h, S, T)$ at one-loop order.

\subsection{Coleman Weinberg potential}

At zero-temperature, the one-loop effective potential can be written as

$V_{\mathrm{eff}}^{T=0}(h, S)=V_{0}+\sum_{i} V_{\mathrm{CW}}^{i}\left(m_{i}^{2}(h, S)\right)$

The Coleman-Weinberg potential is the zero-momentum piece of the zero-temperature effective action, and is a sum of 1PI one-loop diagrams with arbitrary numbers of external $h$ and $S_{0}$ fields and particles $i=\left\{t, W, Z, h, G^{ \pm}, G^{0}, S_{0}, S_{k}\right.$, ... ) running in the loop (where $k>0$ ). Note that we are working in Landau gauge to avoid ghost-compensating terms, which requires including the Goldstone contributions separately, in addition to the massive $W, Z$ bosons. (We discuss issues of gauge invariance in Sect. 2.6.)

The dependence of the $i$ th particle tree-level mass $m_{i}^{2}=$ $m_{i}^{2}(h, S)$ on the VEVs of $h$ and $S$ determines $V_{\mathrm{CW}}$. Summing over all contributions gives [114]:

$V_{C W}^{i}=\frac{1}{2} \int \frac{d^{4} k}{(2 \pi)^{4}} \log \left[k_{E}^{2}+m_{i}^{2}(h, S)\right]$

where e.g. $m_{h}\left(h, S_{0}\right)=-\mu^{2}+3 \lambda h^{2}+\lambda_{H S} S_{0}^{2}$ and $k_{E}$ is the euclidian momentum of particle $i$ in the loop. We adopt the 
dimensional regularization scheme and $\overline{\mathrm{MS}}$ renormalization scheme, with the usual $\epsilon=2-\frac{D}{2}$. This makes one-loop matching more onerous than the on-shell renormalization scheme, but allows for the potential to be RG-improved more easily. The result is

$$
V_{C W}^{i}=\frac{m_{i}^{4}(h, S)}{64 \pi^{2}}\left(-\epsilon-\gamma_{E}+\log 4 \pi+\log \frac{m_{i}^{2}(h, S)}{\mu_{R}^{2}}\right)
$$

(Hereafter we drop the explicit $h, S$ dependence of the masses for brevity.) $\mu_{R}$ is the renormalization scale, and variation of physical observables after matching with different values of $\mu_{R}$ is a common way of assessing the uncertainty of our results due to the finite one-loop perturbative expansion. Adding counterterms and removing divergences yields the familiar expression

$V_{C W}^{i}=(-1)^{F} g_{i} \frac{m_{i}^{4}}{64 \pi^{2}}\left(\log \left[\frac{m_{i}^{2}}{\mu_{R}^{2}}\right]-c_{i}\right)$,

where $F=1(0)$ for fermions (bosons), $c_{i}=\frac{3}{2}\left(\frac{5}{2}\right)$ for scalars/fermions (vectors), and $g_{i}$ is the number of degrees of freedom associated with the particle $i$.

\subsection{Finite temperature}

Finite-temperature quantum field theory (FTQFT) enables the computation of observables, like scalar field vacuum expectation values, in the background of a thermal bath. The corresponding Greens functions can be computed by compactifying time along the imaginary direction, for details see e.g. [18]. To get an intuitive idea of finite-temperature effects on the one-loop effective potential, it is useful to consider integrals of the form

$\int \frac{d k_{0}}{2 \pi} f\left(k_{0}\right)$

where $k_{0}$ is the time-like component of the loop momentum. This can be evaluated in FTQFT as

$$
\int \frac{d k_{0}}{2 \pi} f\left(k_{0}\right) \rightarrow T \sum_{n=-\infty}^{\infty} f\left(k_{0}=i \omega_{n}\right),
$$

where $\omega_{n}=2 n \pi T$ and $(2 n+1) \pi T$ are the Matsubara frequencies for bosons and fermions, respectively. Equation (2.8) can be written in the instructive form:

$$
\begin{aligned}
\int \frac{d k_{0}}{2 \pi} f\left(k_{0}\right) \rightarrow & \int_{-i \infty}^{i \infty} \frac{d z}{4 \pi i}[f(z)+f(-z)] \\
& +\eta \int_{C} \frac{d z}{2 \pi i} n(z)[f(z)+f(-z)],
\end{aligned}
$$

where $\eta= \pm 1$ for bosons/fermions and $n(z)=\left(e^{z / T}-\right.$ $\eta)^{-1}$ are the standard Fermi-Dirac/Bose-Einstein distribu- tion functions, for a particular choice of contour $C$. The first term, which is $n(z)$ independent, is simply the usual zerotemperature loop integral, while the second term is the new contribution from thermal loops in the plasma. This makes effects like thermal decoupling very apparent - if the particle mass is much larger than the temperature, its contribution to the second loop integral will vanish as $n(z) \rightarrow 0$.

Applying this formalism to the one-loop effective potential at finite temperature generalizes Eq. (2.3):

$$
\begin{aligned}
V_{\mathrm{eff}}^{T>0}(h, S, T)= & V_{0}+\sum_{i}\left[V_{\mathrm{CW}}^{i}\left(m_{i}^{2}(h, S)\right)\right. \\
& \left.+V_{\mathrm{th}}^{i}\left(m_{i}^{2}(h, S), T\right)\right]
\end{aligned}
$$

where the second term is the usual Coleman-Weinberg potential, and the third term is the one-loop thermal potential

$$
V_{\mathrm{th}}^{i}\left(m_{i}^{2}(h, S), T\right)=(-1)^{F} g_{i} \frac{T^{4}}{2 \pi^{2}} J_{\mathrm{B} / \mathrm{F}}\left(\frac{m_{i}^{2}(h, S)}{T^{2}}\right)
$$

with thermal functions

$$
J_{\mathrm{B} / \mathrm{F}}\left(y^{2}\right)=\int_{0}^{\infty} d x x^{2} \log \left[1 \mp \exp \left(-\sqrt{x^{2}+y^{2}}\right)\right]
$$

which vanish as $T \rightarrow 0$. Note that $y^{2}$ can be negative. The thermal functions have very useful closed forms in the hightemperature limit,

$$
\begin{aligned}
J_{B}\left(y^{2}\right) \approx & J_{B}^{\text {high }-T}\left(y^{2}\right)=-\frac{\pi^{4}}{45}+\frac{\pi^{2}}{12} y^{2}-\frac{\pi}{6} y^{3} \\
& -\frac{1}{32} y^{4} \log \left(\frac{y^{2}}{a_{b}}\right) \\
J_{F}\left(y^{2}\right) \approx & J_{F}^{\text {high }-T}\left(y^{2}\right)=\frac{7 \pi^{4}}{360}-\frac{\pi^{2}}{24} y^{2} \\
& -\frac{1}{32} y^{4} \log \left(\frac{y^{2}}{a_{f}}\right) \text { for }\left|y^{2}\right| \ll 1,
\end{aligned}
$$

where $a_{b}=\pi^{2} \exp \left(3 / 2-2 \gamma_{E}\right)$ and $a_{f}=16 \pi^{2} \exp (3 / 2-$ $2 \gamma_{E}$ ). This high- $T$ expansion includes more terms, but they do not significantly increase the radius of convergence. With the log term included, this approximation for both the potential and its derivatives is accurate to better than $\sim 10 \%$ even for $m \sim(1-3) \times T$ (depending on the function and order of derivative), but breaks down completely beyond that. The low-temperature limit $\left(\left|y^{2}\right| \gg 1\right)$ also has a useful expansion in terms of modified Bessel functions of the second kind:

$$
\begin{aligned}
J_{B}\left(y^{2}\right) & =\tilde{J}_{B}^{(m)}\left(y^{2}\right)=-\sum_{n=1}^{m} \frac{1}{n^{2}} y^{2} K_{2}(y n) \\
J_{F}\left(y^{2}\right) & =\tilde{J}_{F}^{(m)}\left(y^{2}\right) \\
& =-\sum_{n=1}^{m} \frac{(-1)^{n}}{n^{2}} y^{2} K_{2}(y n) \text { for }\left|y^{2}\right| \gg 1 .
\end{aligned}
$$




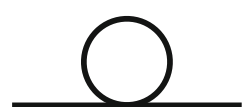

(a)

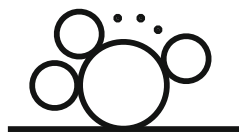

(b)

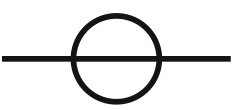

(c)

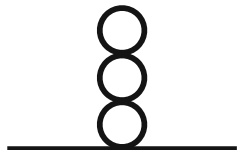

(d)
Fig. 1 Various scalar mass contributions in $\phi^{4}$ theory: a one-loop mass correction, which is quadratically divergent at zero temperature, b higher-loop daisy contributions which are leading order in $T$ and $N$ at high temperature, $\mathbf{c}$ the two-loop "lollipop" contribution which is sub-

This expansion can be truncated at a few terms, $m=2$ or 3 , and yield very good accuracy, but convergence for smaller $\left|y^{2}\right|$ is improved by including more terms.

For negative $m_{i}^{2}$, the effective potential (both zero- and finite-temperature) includes imaginary contributions, which were discussed in [92]. These are related to decay widths of modes expanded around unstable regions of field space, and do not affect the computation of the phase transition. Therefore, we always analyze only the real part of the effective potential.

\subsection{Resummation of the thermal mass: truncated full dressing (TFD)}

The effective potential defined in Eq. (2.10) can be evaluated at different temperatures to find $v_{c}$ and $T_{c}$. The hightemperature expansion of $J_{B}$ already reveals how one particular BSM effect could induce a strong EWPT. If a light boson is added to the plasma with $m_{i}^{2} \sim h^{2}$, then the $-y^{3}$ term in $J_{B}$ will generate a negative cubic term $-h^{3}$ in the effective potential, which can generate an energy barrier between two degenerate vacua. However, the calculation of the effective finite-temperature potential is still incomplete. There is a very well-known problem which must be addressed in order to obtain a trustworthy calculation [81-83].

This can be anticipated from the fact that a symmetry, which is broken at zero temperature, is restored at hightemperature. Thermal loop effects overpower a temperatureindependent tree-level potential. This signals a breakdown of fixed-order perturbation theory, which arises because in FTQFT a massive scalar theory has not one but two scales: $\mu$ and $T$. Large ratios of $T / \mu$ have to be resummed.

To begin discussing thermal mass resummation, let us first consider not our BSM benchmark model but a much simpler $\phi^{4}$ theory with quartic coupling $\lambda$ and $N$ scalar fields which obey a global $O(N)$ symmetry:

$V_{0}=-\frac{1}{2} \mu^{2} \phi_{i} \phi_{i}+\frac{1}{4} \lambda\left(\phi_{i} \phi_{i}\right)^{2}$

(In fact, if we were to ignore fermions and gauge bosons, and set $\lambda=\lambda_{H S}=\lambda_{S}$, our BSM benchmark model would reduce to this case with $N=N_{S}+4$.) leading in $T$ and $N$ to the two-loop daisy, $\mathbf{d}$ the three-loop superdaisy contribution, which is subleading in $T$ but of equal order in $N$ to the three-loop daisy

In FTQFT, the leading order high temperature behavior for diagrams with external scalar lines is directly related to the 0 -temperature superficial degree of divergence $d$. Diagrams which have $d>0$ have a $T^{d}$ high temperature behavior. For $d \leq 0$, there is a linear $T$ dependence. Appropriate factors of the coupling $\lambda, N$ and the tree level mass parameter $\mu$ can be added from vertex counting and dimensional grounds. Therefore, the one-loop scalar mass correction shown in Fig. 1a scales as $\sim N \lambda T^{2}$ to leading order in temperature, which is the "hard thermal loop". The phase transition occurs around the temperature where this thermal mass cancels the treelevel mass at the origin:

$\mu^{2} \sim N \lambda T^{2} \Rightarrow \alpha \equiv N \lambda \frac{T^{2}}{\mu^{2}} \sim 1$.

At $n$-loop order, the leading contribution in temperature to the thermal mass is given by daisy diagrams shown in Fig. 1b:

$\delta m_{\text {n-loop daisy }}^{2} \sim N^{n} \frac{\lambda^{n} T^{2 n-1}}{\mu^{2 n-3}}, \quad n \geq 2$

The ratio of the $n$ to the $n+1$ loop daisy contribution scales as

$\frac{\delta m_{n}^{2}}{\delta m_{n-1}^{2}} \sim \alpha$

which is not parametrically small during the phase transition, causing the perturbative expansion to break down. This can also be understood as an IR divergent contribution (in the high $T$ limit) to the zero mode propagator.

To make the expansion more reliable, it is necessary to resum the thermal mass by replacing the tree-level $m_{\text {tree }}^{2}(\phi)$ in Eq. (2.10) by $m^{2}(\phi)=m_{\text {tree }}^{2}(\phi)+\Pi(\phi, T)$, where in the standard method, $\Pi$ is taken to be the leading contribution in temperature to the one-loop thermal mass. For scalars this can be obtained by differentiating $V_{\text {th }}$ with respect to $\phi$ :

$\Pi \sim \lambda T^{2}+\cdots$

The ellipses represent subleading contributions in both the high-temperature expansion and coupling order, which are neglected.

This substitution automatically includes daisy contributions to all orders in the effective potential. The largest con- 
tributions which are not included are the two-loop "lollipop" diagrams shown in Fig. 1c, scaling as $\lambda^{2} T^{2} N$, and the threeloop superdaisy shown in Fig. 1d) scaling as $\lambda^{3} T^{4} N^{3} / \mu^{2}$. Reliability of the perturbative expansion with the above thermal mass substitution requires

$\lambda \ll 1, \quad \beta \equiv \frac{\lambda T N}{\mu} \ll 1$.

These are obtained by requiring the ratio of the one-loop thermal mass to the sunset and the ratio of the two-loop daisy to the three-loop daisy to be small.

To illustrate how this resummation procedure is implemented in most BSM calculations, let us again turn to our $S M+N_{S} \times S$ benchmark model. The "dressed" effective potential is given by

$$
\begin{aligned}
V_{\mathrm{eff}}^{\mathrm{dressed}}(h, S, T)= & V_{0}+\sum_{i}\left[V_{\mathrm{CW}}^{i}\left(m_{i}^{2}(h, S)+\Pi_{i}\right)\right. \\
& \left.+V_{\mathrm{th}}^{i}\left(m_{i}^{2}(h, S)+\Pi_{i}, T\right)\right],
\end{aligned}
$$

where

$$
\begin{aligned}
\Pi_{h}(0) & =\Pi_{G}(0) \\
& =T^{2}\left(\frac{3}{16} g^{2}+\frac{1}{16} g^{\prime 2}+\frac{1}{4} \lambda_{t}^{2}+\frac{1}{2} \lambda+\frac{N_{S}}{12} \lambda_{H S}\right) \\
\Pi_{S}(0) & =T^{2}\left(\frac{1}{3} \lambda_{H S}+\frac{N_{S}+2}{12} \lambda_{S}\right) \\
\Pi_{G B}^{L}(0) & =\frac{11}{6} T^{2} \operatorname{diag}\left(g^{2}, g^{2}, g^{2}, g^{\prime 2}\right)
\end{aligned}
$$

and $\Pi_{G B}^{L}$ is added only to the longitudinal gauge boson masses squared in the gauge basis, which are then diagonalized. Gauge symmetry suppresses thermal contributions to the transverse mode [85]. Note that while fermions receive thermal mass, there are no zero modes $\omega_{n}=(2 n+1) \pi T$, and as a result no IR divergences appear in the fermion propagator.

As we explain in Sect. 3, substituting $m_{\text {tree }}^{2}+\Pi$ directly into the effective potential is called full dressing (FD). Since the thermal mass $\Pi$ is explicitly evaluated only to leading order in the high-temperature expansion, we refer to this resummation procedure as truncated full dressing (TFD). TFD is the standard approach for BSM calculations of the EW phase transition.

If $V_{\mathrm{th}}^{i}$ is expanded using the high-Temperature approximation of Eq. (2.13), the field dependent terms in logs cancel between $V_{\mathrm{CW}}^{i}$ and $V_{\mathrm{th}}^{i}$. The $y^{2}$ term gives an overall contribution proportional to $T^{2} \Pi_{i}$, which is field-independent when using only the leading-order contribution to $\Pi_{i}$ in temperature. This just leaves the $y^{3}$ term, which can be captured by adding $V_{\text {ring }}^{i}$ :

$$
\begin{aligned}
V_{\mathrm{eff}}^{\mathrm{dressed}}(h, S, T)= & V_{0}+\sum_{i}\left[V_{\mathrm{CW}}^{i}\left(m_{i}^{2}(h, S)\right)\right. \\
& \left.+V_{\mathrm{th}}^{i}\left(m_{i}^{2}(h, S), T\right)+V_{\text {ring }}^{i}\left(m_{i}^{2}(h, S), T\right)\right],
\end{aligned}
$$

where

$$
\begin{aligned}
V_{\text {ring }}^{i}\left(m_{i}^{2}(h, S), T\right)= & -\frac{g_{i} T}{12 \pi}\left(\left[m_{i}^{2}(h, S)+\Pi_{i}\right]^{3 / 2}\right. \\
& \left.-\left[m_{i}^{2}(h, S)\right]^{3 / 2}\right) .
\end{aligned}
$$

Adding $V_{\text {ring }}^{i}$ amounts to resumming the IR-divergent contributions to the Matsubara zero mode propagator. It is tantamount to performing the $m_{i}^{2} \rightarrow m_{i}^{2}+\Pi_{i}$ replacement in the full effective potential, under the assumption that only the thermal mass of the zero mode matters, which is equivalent to making a high-temperature approximation.

This is the version of the finite-temperature effective potential used in most BSM calculations. In some cases, Eq. (2.23) is used but with the full finite-temperature $V_{\mathrm{th}}^{i}$ instead of the high-T expansion. This is more accurate when $m_{i}$ is comparable to the temperature, but in that case the assumptions that justify using $V_{\text {ring }}$ are explicitly violated, and Eq. (2.21) is the more consistent choice. In practice, there is not much numerical difference between these two recipes. As we discuss in Sect. 2.6, all of these TFD calculations have problems arising from using only the leading-order contribution of $\Pi_{i}$ in temperature.

\subsection{Types of electroweak phase transitions}

It is well-known that in the SM for $m_{h} \gtrsim 70 \mathrm{GeV}$, the EWPT is not first-order [22,23]. To make the PT first order, new physics effects have to be added to the SM to generate an energy barrier between two degenerate vacua at $T=T_{c}$. These BSM scenarios can be broadly classified into a few classes (see also [97,115]) based on the origin of the barrier between the two degenerate vacua. These are phase transitions driven by thermal effects, tree-level renormalizable effects, loop effects at zero-temperature and nonrenormalizable operators. Note that our simple BSM benchmark model realizes the first three of these mechanisms. Rigorous study of the fourth mechanism will require the updated thermal resummation procedure we present in this paper.

\section{$P T$ driven by BSM thermal effects}

It is possible that BSM bosonic degrees of freedom are present in the plasma. If they have the right mass and coupling to the SM Higgs, they can generate an energy barrier to make the PT strongly first order. Schematically, this can be understood as follows. If the boson(s) have tree-level mass $m_{i}^{2}=\mu_{S}^{2}+\lambda_{H S} h^{2}$, the effective potential of Eq. (2.23) contains a term of the form 


$$
-\frac{T}{12 \pi}\left[\mu_{S}^{2}+\lambda_{H S} h^{2}+\Pi_{S}\right]^{3 / 2}
$$

If this term is dominated by the $h$-dependent piece at $T=$ $T_{c}$, the resulting $\sim-\left(\lambda_{H S}\right)^{\frac{3}{2}} h^{3} T$ negative cubic term can generate an energy barrier between two degenerate minima and catalyze a strong first order PT.

In order for this cubic term to be manifest, it is required that $\mu_{S}^{2}+\Pi_{S} \ll \lambda_{H S} v_{c}^{2}$. In the SM the $W$ and $Z$ bosons generate a cubic term, but their contribution is too small to make the SM EWPT first order. This can be enhanced in BSM scenarios by a partial cancellation between the new boson's thermal mass and a negative bare mass at $T=T_{c}$.

This scenario was long regarded as one of the most promising avenues for EWBG, because light stops in supersymmetry could serve as these new bosonic degrees of freedom (DOF) [50-60]. Higgs coupling measurements have since excluded that possibility for the MSSM [61,62] and general models with colored scalars $[63,64]$. Other scenarios, including the $\mathrm{SM}+N_{S} \times S$ benchmark model we explore here, can easily realize this possibility $[63,64]$.

The mass of these light BSM bosonic DOF cannot significantly exceed $T_{c} \sim \mathcal{O}(100 \mathrm{GeV})$ to ensure their thermal contributions are unsuppressed. This makes such EWBG scenarios prime candidates for discovery at the LHC, and possibly future colliders. It is therefore of paramount importance to robustly correlate the predicted collider signatures with the regions of parameter space which allow for a strong phase transition.

This mechanism relies on a partial cancellation between a zero-temperature mass and a thermal mass. However, in the standard calculation, the thermal mass is computed only to leading order in the high-T expansion. This is troubling, since (a) even within the high-T expansion, subleading terms in the expansion can change the thermal mass by $\mathcal{O}(40 \%)$ or more [116], and (b) the thermal mass should decrease for nonzero Higgs expectation values, since the bosons become heavier as $h \rightarrow v_{c}$ and partially decouple from the plasma. This can affect the electroweak phase transition, and the corresponding predictions for collider observables from a strong EWPT. Addressing this issue will be one of the major goals of our work

\section{PT driven by tree-level renormalizable effects}

It is possible to add new scalars to the SM Higgs potential, see e.g. [24,34,37,117]. In that case, the tree-level structure of the vacuum can be modified. For example, it is possible for the universe to first transition to a nonzero VEV of an additional singlet, only to transition to another vacuum with a nonzero Higgs VEV at a lower temperature. It is also possible for the Higgs to mix with new DOF (i.e. both the Higgs and the new DOF acquire VEVs in our vacuum). In that case, the tree-level potential can have a barrier between the origin and the EWSB minimum, result- ing in a strong one-step phase transition at finite temperature.

These tree-driven one- or two-step PTs can easily be very strongly first order, but can also cause runaway bubbles, which are incompatible with sufficient BAU generation [118]. On the other hand, the strong nature of these PTs might make them discoverable by future gravitational wave observations [113]. It is therefore important to understand which regions of parameter space are associated with these types of phase transitions.

An intriguing version of the two-step EWBG scenario is possible when a triplet scalar is added to the SM [47-49]. In that case, the baryon asymmetry can be created in the first transition to the triplet-VEV-phase, and preserved in the second transition to the doublet-VEV phase which the universe inhabits at zero temperature.

\section{PT driven by loop effects at zero temperature}

New degrees of freedom with sizable couplings to the Higgs can generate non-analytical contributions to $V_{\mathrm{CW}}$ at zero temperature which "lift" the local $h=v$ minimum to a higher potential relative to the origin, compared to the SM. With this shallower potential well, SM $Z$ and $W$ boson thermal contributions can be strong enough to generate a cubic potential term at finite temperature, resulting in a strong PT. This was recently discussed in the context of future collider signatures by [111], and we will generalize their phenomenological results in this paper.

\section{PT driven by non-renormalizable operators}

The previous two phase transition classes are primarily associated with the zero-temperature effects of BSM degrees of freedom on the Higgs potential. If these states are sufficiently heavy, it might be reasonable to parametrize some of their effect in an EFT framework by adding a set or nonrenormalizable operators to the SM Higgs potential. This was used to correlate Higgs self-coupling deviations with a strong EWPT [90-92].

While EFT analyses are useful for analyzing broad classes of new physics effects, their construction and validity at finite temperature is not well-understood. ${ }^{1}$ At zero-temperature experiments, like mono-energetic collisions with energy $E$, the effects of perturbatively coupled particle with mass $m$ can be well described by an EFT if $m / E>1$. This is not the case in a plasma, where the heavy state can be directly excited even if $m / T$ is larger than unity, generating sizable thermal loop contributions. Furthermore, EFTs are problematic when studying phase transitions, since the spectrum which is integrated out changes between the two

\footnotetext{
1 The authors of [119] studied the agreement between a singlet extension of the SM and the corresponding EFT, but since TFD was used decoupling effects could not be correctly modeled.
} 
vacua. Finally, the agreement between a full theory including heavy states and an EFT description cannot presently be studied reliably. This is because in the TFD thermal mass resummation procedure, the effects of new particles in the full theory on light scalar thermal masses never decouples since $\Pi \sim T^{2}$ is independent of contributing particle masses. The non-decoupling of high mass DOFs in the full theory calculation is clearly unphysical, preventing us from understanding the EFT's radius of convergence in field space and temperature. This provides another strong motivation for treating thermal masses more carefully.

2.6 Problems with the standard one-loop TFD calculation of the phase transition

There are a few ways in which the standard calculation with TFD thermal mass resummation, as outlined above, is incomplete and can be extended.

1. Resumming Goldstones: At zero temperature, SM Goldstone contributions must be resummed to eliminate the unphysical divergence in the derivatives of $V_{\mathrm{CW}}$ when their masses at tree-level are zero $[120,121]$. The numerical effects of the Goldstone contributions, once resummed, are small, so we can deal with this by not including Goldstones in the loop calculations of certain couplings. In the $\overline{\mathrm{MS}}$ scheme this is not a (numerical) problem as long as the tree-level Higgs VEV is somewhat shifted from the loop-level Higgs VEV.

2. Gauge dependence: Since the potential is derived from the gauge-dependent 1PI effective action, $v_{c}$ is not a gauge-independent quantity. In the standard Landaugauge-fixed calculation, we compute $v_{c} / T_{c}$ as a proxy for the sphaleron energy in the broken phase (which is gauge independent), and the requirement that $v_{c} / T_{c}>0.6$ is understood to be an approximate minimal necessary condition for EWBG to be plausible.

A fully gauge-independent calculation of $T_{c}$ and the sphaleron energy would make the calculation more reliable. This problem was considered by the authors of [80] in the high-temperature approximation. The gauge dependent potential without any thermal mass resummation is

$$
\begin{aligned}
V_{\mathrm{eff}}^{T>0}(h, S, T ; \xi)= & V_{0}+\sum_{i}\left[V_{\mathrm{CW}}^{i}\left(m_{i}^{2}(h, S ; \xi)\right)\right. \\
& \left.+V_{\mathrm{th}}^{i}\left(m_{i}^{2}(h, S ; \xi), T\right)\right]
\end{aligned}
$$

where $\xi$ is the gauge parameter. Consider the gauge dependence of the third term $V_{\mathrm{th}}^{i}$ :

$$
\begin{aligned}
V_{\text {th }}^{i}= & \frac{T^{4}}{2 \pi^{2}}\left[\sum_{\text {scalar }, i} J_{B}\left(\frac{m_{i}^{2}(h, S ; \xi)}{T^{2}}\right)\right. \\
& \left.+3 \sum_{\text {gauge, } a} J_{B}\left(\frac{m_{a}^{2}(h)}{T^{2}}\right)-\sum_{\text {gauge, } a} J_{B}\left(\frac{\xi m_{a}^{2}(h)}{T^{2}}\right)\right],
\end{aligned}
$$

where $m_{i}^{2}(h ; \xi)=m_{i}^{2}(h)+\xi m_{a}^{2}$, and we have dropped fermion contributions which do not contain any gauge dependence. In the high-temperature expansion and for small $\xi$, the $\xi$-dependent contribution of DOF $i$ charged under the gauge symmetry is

$$
\begin{aligned}
V_{\mathrm{th}}^{i}[\xi]= & \frac{T}{2 \pi^{2}}\left(m_{i}^{3}(h ; \xi)-\xi m_{a}^{3}(h)\right) \\
& +\frac{m_{i}^{4}(h ; \xi)-\xi m_{a}^{4}(h)}{64 \pi^{2}} \log \frac{\mu_{R}^{2}}{T^{2}} .
\end{aligned}
$$

Note that the $\mathcal{O}\left(T^{2}\right)$ term, and hence the dominant contribution to the thermal mass, is gauge-independent. This means that $T_{c}$ only has a small gauge dependence, confirmed by [80] for small values of $\xi$. Furthermore, for singlet extensions the new contributions to the potential which drive the strong phase transition are by definition gauge-independent. Therefore we do not deal with the issue of gauge dependence here and proceed with the standard Landau gauge-fixed calculation. Certainly, further work is needed to construct a fully gaugeindependent general calculation of the strength of the electroweak phase transition, and to understand how sensitive the results of a gauge-fixed calculation are to the choice of gauge parameter.

3. RG-improvement: the convergence of the one-loop effective potential can be improved by using running couplings with 2-loop RGEs. This is independent of other improvements to the calculation and is most important when the theory contains sizable mass hierarchies. We will not discuss it further here.

4. Higher-loop corrections: it is possible to evaluate higherloop contributions to the zero- and finite-temperature effective potential, such as the 2-loop lollipop that is not included via thermal mass resummation. Alternatively, estimates of these contributions can be used to determine whether the one-loop expansion is reliable. In our BSM calculations we will carefully do the latter, using high- $T$ approximations for the relevant diagrams.

5. Consistent thermal mass resummation: In the standard Truncated Full Dressing calculation outlined above, the effective finite-temperature Higgs potential is computed by inserting the truncated thermal masses $\Pi_{i} \sim T^{2}$ into the one-loop potential as shown in Eq. (2.21). This is also called "resumming hard thermal loops", since it amounts 
to resumming only the contribution to the Matsubara zero mode propagator. This is indeed correct, if those contributions dominate the sum of diagrams, which is the case in the extreme limit of the high-temperature approximation.

Early calculations that used this approximation [81$83,122]$ were interested mainly in the restoration of electroweak symmetry at high temperature. Determining $T_{c}$ with reasonable accuracy only requires considering the origin of the Higgs potential where the top and gauge boson masses are entirely dominated by thermal effects. In this case, the truncated high- $T$ expansion for $\Pi_{i}$ is justified, though there are significant deviations which arise from subleading terms in the high-temperature expansion even at the origin.

However, when studying the strong first-order phase transition and computing $v_{c}$, we have to deal with finite excursions in field space which by definition are comparable to the temperature. For $h \sim T$, masses which depend on the Higgs VEV due to a Higgs coupling strong enough to influence the PT cease to be small at tree-level compared to thermal effects, and should start decoupling smoothly from the plasma. The resulting $h$-dependence of $\Pi_{i}(h, T)$ is therefore important. This is especially the case when the strong phase transition is driven by light bosons in the plasma, and therefore reliant on the partial cancellation between a zero-temperature mass and a thermal mass correction. Obtaining correct collider predictions of a strong EWPT requires going beyond the TFD scheme.

As mentioned previously, the high- $T$ thermal mass resummation is also incompatible with any EFT framework of computing the electroweak phase transition, since in this approximation the contribution of heavy degrees of freedom to thermal masses does not decouple. This confounds efforts to find a consistent EFT description of theories at finite temperature. Since EFTs are such a powerful tool for understanding generic new physics effects at zero temperature, rigorously generalizing their use to finite temperature is highly motivated.

We will concentrate on ameliorating the problems associated with TFD thermal mass resummation. Some of the necessary components exist in the literature. It is understood that a full finite-temperature determination of the thermal mass can give significantly different answers from the high$T$ expansion for the thermal mass [116]. This was partially explored, to subleading order in the high- $T$-expansion, for $\phi^{4}$ theories [84-89], but never in a full BSM calculation, without high-temperature approximations.

We will perform a consistent (to superdaisy order) finitetemperature thermal mass computation by numerically solving the associated gap equation and resumming its contribu- tions in such a way as to avoid miscounting important higherloop contributions. Since we are interested in the effect of adding new BSM scalars to the SM in order to generate a strong EWPT, we will be performing this procedure in the scalar sector only. We now explain this in the next section.

\section{Formal aspects of finite-temperature mass resummation}

As outlined above, in a large class of BSM models a strong EWPT is generated due to new weak-scale bosonic states with large couplings to the Higgs. A near-cancellation between the new boson's zero-temperature mass and thermal mass can generate a cubic term in the Higgs potential, which generates the required energy barrier between two degenerate minima $h=0, v_{c}$ at $T=T_{c}$. To more accurately study the phase transition (and correlated experimental predictions) in this class of theories, we would like to be able to compute the thermal masses $\Pi_{i}$ of scalars $i$ beyond the hard thermal loop approximation used in TFD. In other words, rather than resumming only the lowest-order thermal mass in the high-temperature expansion, $\Pi_{i} \sim T^{2}$, we aim to compute and resum the full field- and temperature-dependent thermal mass $\delta m_{i}(h, T)$, with individual contributions to $\delta m_{i}$ accurately vanishing as degrees of freedom decouple from the plasma. We also aim to formulate this computation in such a way that it can be easily adapted for other BSM calculations, and the study of Effective Field Theories at finite Temperature.

A straightforward generalization of the TFD calculation might be formulated as follows. The finite-temperature scalar thermal masses can be obtained by solving a one-loop gap equation of the form

$\delta m_{i}^{2}=\sum_{j} \partial_{i}^{2}\left(V_{\mathrm{CW}}^{j}+V_{\mathrm{th}}^{j}\right)$,

where $\partial_{i}$ represents the derivative with respect to the scalar $i$. The hard thermal loop result $\delta m_{i}^{2} \sim T^{2}$ is the solution at leading order in large $T / m$. To obtain the finite-temperature thermal mass, we can simply keep additional orders of the high$\mathrm{T}$ expansion, or indeed use the full finite-temperature thermal potential of Eqs. $(2.11,2.12)$ in the above gap equation. In the latter case, the equation must be solved numerically. Once a solution for $\delta m_{i}^{2}(h, T)$ is obtained, we can obtain the improved one-loop potential by substituting $m_{i}^{2} \rightarrow m_{i}^{2}+\delta m_{i}^{2}$ in $V_{\mathrm{CW}}+V_{\text {th }}$ as in Eq. (2.21).

Solving this gap equation, and substituting the resulting mass correction into the effective potential itself, is called full dressing [84-86]. This procedure is physically intuitive, but it is not consistent. Two-loop daisy diagrams, which can be important at $T=T_{c}$, are miscounted [88,89]. 
An alternative construction involves substituting $m_{i}^{2} \rightarrow$ $m_{i}^{2}+\delta m_{i}^{2}$ in the first derivative of the effective potential. This tadpole resummation, called partial dressing, was outlined for $\phi^{4}$ theories in [87]. The authors claim that PD correctly counts daisy and superdaisy diagrams to higher order than FD.

There appears to be some confusion in the literature as to whether FD or PD is correct [84-89], but we have repeated the calculations of [87], and confirm their conclusions. Partial dressing (a) consistently resums the most dominant contributions in the high-temperature limit, where resummation is important for the convergence of the perturbative finitetemperature potential, (b) works to higher order in the hightemperature expansion, including the important log term, to correctly model decoupling of modes from the plasma, and (c) is easily adaptable to general BSM calculations. We review the important features of PD below and then outline how to generalize this procedure to numerically solve for the thermal mass at finite temperature. sion. The first derivative of the one-loop effective potential $V_{1}=V_{\mathrm{CW}}+V_{\mathrm{th}}$, see Eqs. (2.6), (2.11) and (2.13), without any thermal mass resummation, is

$$
\frac{\partial V_{1}}{\partial \phi}=V_{1}^{\prime}=(6 \lambda \phi)\left[\frac{T^{2}}{24}-\frac{T m}{8 \pi}-\frac{m^{2} L}{32 \pi^{2}}\right]
$$

where $L=\ln \frac{\mu_{R}^{2}}{T^{2}}-3.9076$ and the $\log$-term arises from a cancellation between the zero- and finite-temperature potential. The tree-level scalar mass is $m^{2}=-\mu^{2}+\frac{\lambda}{2} \phi^{2}$. Differentiating this once again will yield the one-loop thermal mass shown in Fig. 2, as well as an electron-self-energytype diagram for $\phi \neq 0$ that descends from loop corrections to the quartic coupling. During the phase transition, $\alpha \equiv N \lambda T^{2} / m^{2} \approx 1$, requiring daisies to be resummed. This is evident in Fig. 2 from the fact that subsequent terms in each family of diagrams (Daisy, Super-Daisy, Lollipop and Sunset) is related to the previous one by a factor of $\alpha$.

The second derivative of the one-loop potential defines a gap equation, which symbolically can be represented as

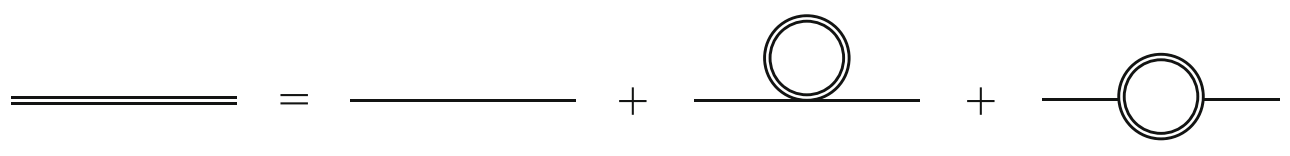

\subsection{Tadpole resummation in $\phi^{4}$ theories}

To explore the correct resummation procedure we first study a $\phi^{4}$ theory with $N$ real scalars obeying an $O(N)$ symmetry. This can then be generalized to the BSM theories of interest for EWBG. The tree-level potential is

$V_{0}=-\frac{1}{2} \mu^{2} \phi_{i} \phi_{i}+\frac{1}{4} \lambda\left(\phi_{i} \phi_{i}\right)^{2}$.

Without loss of generality, assume that all excursions in field space are along the $\phi_{0}$ direction.

Resummation of the thermal mass is required when hightemperature effects cause the fixed-order perturbation expansion to break down. We are therefore justified in using the high- $T$ expansion to study the details of the thermal mass resummation procedure and ensure diagrams are not miscounted. Conversely, when $T \sim m$, there is no mismatch of scales to produce large ratios that have to be resummed. In this limit, the thermal mass will be less important, but should decouple accurately, and the resummed calculation should approach the fixed-order calculation. We now review how the PD procedure outlined in [87] achieves both of these objectives.

\subsubsection{Partial dressing results for $N=1$}

We start by summarizing the main result of [87], which studied the $N=1 \phi^{4}$ theory in the high-temperature expan- where double-lines represent improved propagators with the resummed mass $M$, while single-lines are un-improved propagators with the tree-level mass $m$. Algebraically, this gap equation is obtained by substituting $M^{2}$ into the second derivative of the one-loop effective potential:

$$
\begin{aligned}
M^{2}= & m^{2}+\left.V_{1}^{\prime \prime}\right|_{m^{2} \rightarrow M^{2}} \\
\Rightarrow \quad M^{2}= & m^{2}+\frac{\lambda T^{2}}{4}-\frac{3 \lambda T M}{4 \pi} \\
& -\frac{3 \lambda M^{2} L}{16 \pi^{2}}-\zeta\left[\frac{9 \lambda^{2} \phi^{2} T}{4 \pi M}+\frac{9 \lambda^{2} \phi^{2}}{8 \pi^{2}}\right]
\end{aligned}
$$

where we have inserted a factor $\zeta=1$ for reasons which will be made clear below. The PD procedure involves resumming these mass corrections by substituting $m^{2} \rightarrow M^{2}$ in the first derivative of the potential Eq. (3.3) rather than the potential itself. The potential is then obtained by integrating with respect to $\phi$ :

$\left.V_{1}^{p d} \equiv \int d \phi V_{1}^{\prime}\right|_{m^{2} \rightarrow M^{2}(\zeta=1)}$.

By expanding the above in large $T$, one can show that $V_{1}^{p d}$ correctly includes all daisy and super-daisy contributions shown (in the form of mass contributions) in Fig. 2, to both leading, sub-leading and log-order in temperature.

This partial dressing procedure does make one counting mistake, which is that all the sunset diagrams in Fig. 2, starting at 2-loop order and nonzero for $\phi \neq 0$, are included with an overall multiplicative pre-factor of $3 / 2$. This can be 


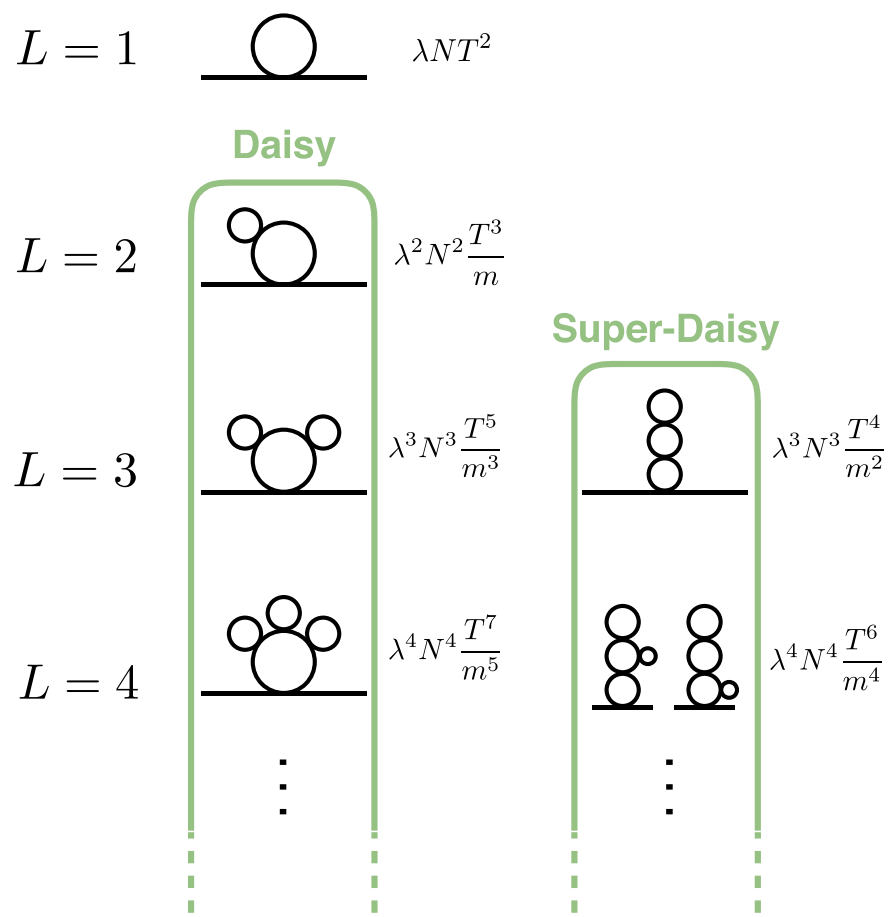

Fig. 2 Complete set of 1- and 2- loop contributions to the scalar mass, as well as the most important higher loop contributions, in $\phi^{4}$ theory. The scaling of each diagram in the high-temperature approximation is indicated, omitting symmetry- and loop-factors. Diagrams to the right of the vertical double-lines only contribute away from the origin

fixed by changing $\zeta$ in the gap equation (3.4) from 1 to $2 / 3$, resulting in the one-loop effective potential

$\left.V_{1}^{p d_{2 / 3}} \equiv \int d \phi V_{1}^{\prime}\right|_{m^{2} \rightarrow M^{2}(\zeta=2 / 3)}$

Finally, non-daisy type diagrams, most importantly the two-loop lollipop in Fig. 2 and its daisy-dressed descendants, are by definition not included in this one-loop resummed potential. However, in the high-temperature limit they can be easily included by adding the explicit expression for the lollipoop loop tadpole (one external $\phi$ line, hence the name),

$V_{2}^{\prime} \supset V_{\ell}^{\prime}=(6 \lambda \phi) \frac{\lambda T^{2}}{32 \pi^{2}}\left[\log \frac{m^{2}}{T \mu_{R}}+1.65\right]$

with the same $m^{2} \rightarrow M^{2}(\zeta=2 / 3)$ substitution:

$V_{1}^{p d_{2 / 3}+\ell} \equiv \int d \phi\left[V_{1}^{\prime}+V_{\ell}^{\prime}\right]_{m^{2} \rightarrow M^{2}(\zeta=2 / 3)}$

$\zeta=1$ can also be used, in which case 2-loop sunsets are not corrected.) This effective potential includes all daisy, superdaisy, sunset and lollipop contributions correctly, and is therefore correct to three-loop superdaisy order.

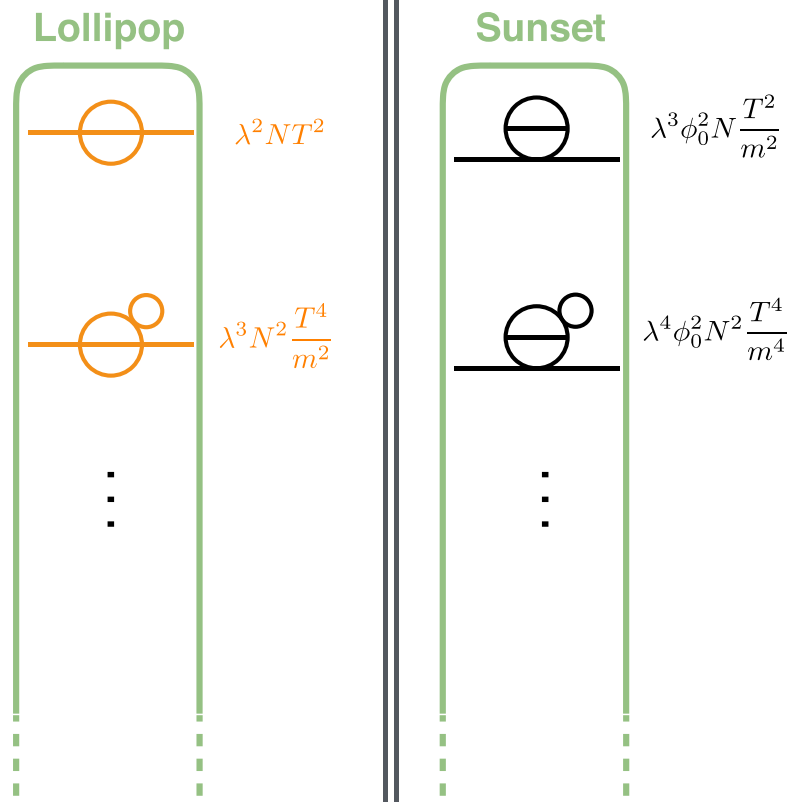

when $\langle\phi\rangle=\phi_{0}>0$. We do not show contributions which trivially descend from e.g. loop-corrected quartic couplings. Lollipop diagrams (in orange) are not automatically included in the resummed one-loop potential

\subsubsection{Comparing resummation schemes}

We have verified that the above results generalize to $O(N)$ $\phi^{4}$ theories, with $N>1$. As mentioned above, at temperatures near the phase transition the parameter $\alpha \equiv N \lambda T^{2} / \mathrm{m}^{2}$ is $\approx 1$, necessitating resummation. To compare different resummation approaches, let us first define which parameters are required to be small for the improved perturbative expansion to converge. Zero-temperature perturbation theory requires

$N \lambda \ll 1$

(where the above equation, and other inequalities of its type, typically contain loop- and symmetry-factors which we usually suppress). Satisfying Eq. (3.9) means that during the phase transition, in regions of field space where $\alpha \approx 1$, the high-temperature expansion (whether in $T / m$ or $T / M$ ) is usually valid. In order for the series of high-temperature contributions to converge, the parameter $\beta$ must also be small:

$\beta \equiv N \lambda \frac{T}{m}=\alpha \sqrt{N \lambda} \ll 1$.

An easy way to see this is to examine Fig. 2. Let us call the mass contribution of the one-loop $\sim T^{2}$ diagram $\delta m_{1}$, and the total contributions of the daisy, super-daisy, lollipop and suset 
family of diagrams $\delta m_{D}^{2}, \delta m_{S D}^{2}, \delta m_{L L}^{2}, \delta m_{S S}^{2}$ respectively. After resummation in $\alpha$, we obtain ${ }^{2}$

$$
\begin{aligned}
\delta m_{1}^{2} \sim \lambda N T^{2} \quad \delta m_{D}^{2} \sim \lambda^{2} N^{2} \frac{T^{3}}{m} \quad \delta m_{S D}^{2} \sim \lambda^{3} N^{3} \frac{T^{4}}{m^{2}} \\
\delta m_{L L}^{2} \sim \lambda^{2} T^{2} N \quad \delta m_{S S}^{2} \sim \lambda^{3} \phi_{0}^{2} N \frac{T^{2}}{m^{2}}
\end{aligned}
$$

Making use of $\alpha \approx 1$, these contributions arrange themselves in order of size:

$$
\begin{aligned}
\frac{\delta m_{D}^{2}}{\delta m_{1}^{2}} \sim \frac{\delta m_{S D}^{2}}{\delta m_{D}^{2}} \sim \alpha \frac{m}{T}=\beta \\
\frac{\delta m_{L L}^{2}}{\delta m_{D}^{2}} \sim \frac{1}{N} \frac{m}{T}=\frac{1}{\alpha} \frac{1}{N} \beta \\
\frac{\delta m_{S S}^{2}}{m_{D}^{2}} \sim \frac{\alpha}{N^{2}} \frac{\phi_{0}^{2}}{T^{2}} \frac{m}{T}=\frac{\phi_{0}^{2}}{T^{2}} \frac{1}{N^{2}} \beta
\end{aligned}
$$

Clearly $\beta$ is the relevant expansion parameter which has to be small for the series to converge. Furthermore, in terms of $\beta$, both the lollipop and sunset diagrams are of the same order as the superdaisy family, but with additional suppression factors of $1 / N .^{3}$ (In our regime of interest, $\phi_{0}$ is usually not much larger than $T$, so the sunsets are subdominant or at most comparable to the lollipop and superdaisy.)

We can now carefully compare different resummation approaches. The partial dressing procedure, with the lollipop correction and the additional sunset contribution, is accurate to $\mathcal{O}\left(\beta^{2}\right)$. Since tree- and loop-contributions are of similar size near the phase transition we compare all sub-leading contributions to the unimproved one-loop thermal mass $\delta m_{1}^{2}$. Relative to $\delta m_{1}^{2}$, the size of neglected zero-temperature contributions and non-daisy contributions at three-loop order are

$(N \lambda)^{2}$ and $\beta^{3} \sim(N \lambda)^{3 / 2}$,

respectively.

The alternative full dressing procedure [84-86] involves solving the same gap equation as for partial dressing, but substituting $m^{2} \rightarrow M^{2}$ in the potential $V_{1}$ instead of its first derivative. This essentially dresses up both the propagator and the cubic coupling in the potential. ${ }^{4}$

The authors of [87] demonstrate that FD miscounts daisies and super-daisies (starting at the 2-loop level), does not

\footnotetext{
2 Note that in the individual diagrams of Fig. 2, the un-improved treelevel mass $m$ is used in the propagator. The entire e.g. lollipop series can be obtained by evaluating the leading diagram with the daisy-improved mass $M$.

${ }^{3}$ Due to the different symmetry factors of the lowest-order superdaisy and lollipop diagrams, the corresponding $N$-suppression is not numerically significant for $N \lesssim 10$.

4 This inspires the name we use for the standard thermal mass resummation as reviewed in Sect. 2.4. Since it involves computing the mass correction to leading order in high temperature $\Pi \sim T^{2}$ and substituting $m^{2}+\Pi$ into the effective potential, we call it truncated full dressing, even though at $\mathcal{O}\left(T^{2}\right)$ there is no actual difference between FD and PD.
}

include sunset contributions, and includes lollipop contributions but with a wrong prefactor and without the logdependence of Eq. (3.7), which arises from neglecting internal loop momenta (as expected in a resummation procedure which does not explicitly calculate multi-loop diagrams). We have confirmed their results. Therefore, ignoring the incorrect accounting of the lollipop which vanishes at the origin, the error terms of a PD calculation are

$(N \lambda)^{2}$ and $\beta \sim(N \lambda)^{1 / 2}$,

The standard BSM calculation is even worse, since TFD only uses the leading-order thermal mass, leading to possible error terms

$(N \lambda)^{2}, \quad \beta \sim(N \lambda)^{1 / 2}$ and $\frac{m}{T}$.

The advantages of partial dressing, compared to the truncated (or un-truncated) full dressing procedure, are clear, especially for phase transitions driven by BSM thermal effects, where the $m / T$ error in Eq. (3.16) can be significant.

\subsection{A general resummation procedure for BSM theories}

We now discuss how to adapt partial dressing for efficient calculation of the phase transition in general BSM theories. We will limit ourselves to phase transitions along the Higgs direction, briefly discussing other cases in the next subsection.

Since partial dressing avoids miscounting of the most important thermal contributions at all orders in the high$\mathrm{T}$ expansion, including the log-term, it can be explicitly applied to the finite-temperature regime. In regions where $\alpha \approx 1$, the high-temperature expansion is valid, and resummation will be properly implemented. This smoothly interpolates to the regime where masses are comparable to temperature, eliminating the separation of scales and making the fixed-order calculation reliable again, with finite-temperature effects decoupling correctly as the mass is increased. Therefore, for a given set of mass corrections $\delta m_{i}^{2}$ for gauge bosons and scalars $i$, we define our effective potential along the $h$ direction by substituting the mass corrections into the first derivative of the loop potential:

$$
\begin{aligned}
V_{\mathrm{eff}}^{\mathrm{pd}}(h, T)= & V_{0}+\sum_{i} \int d h\left[\frac{\partial V_{\mathrm{CW}}^{i}}{\partial h}\left(m_{i}^{2}(h)+\delta m_{i}^{2}(h, T)\right)\right. \\
& \left.+\frac{\partial V_{\mathrm{th}}^{i}}{\partial h}\left(m_{i}^{2}(h)+\delta m_{i}^{2}(h, T), T\right)\right]
\end{aligned}
$$

Note that $V_{\text {th }}$ is not (necessarily) expanded in high- or lowtemperature.

Next, how do we obtain the mass corrections $\delta m_{i}^{2}$ ? We will concentrate on cases, like our $S M+N_{S} \times S$ benchmark model, in which the dominant effect of new physics 
on the phase transition comes from an expanded scalar sector. Therefore, we will retain use of the $\mathcal{O}\left(T^{2}\right)$ gauge boson thermal masses of Eq. (2.22), and set

$\delta m_{G B}^{2}(h)=\Pi_{G B}(0)$

For the scalar mass corrections we numerically solve a set of coupled gap equations at each different value of $h$ and $T$ :

$$
\begin{array}{r}
\delta m_{\phi_{j}}^{2}(h, T)=\sum_{i}\left[\frac{\partial^{2} V_{\mathrm{CW}}^{i}}{\partial \phi_{j}^{2}}\left(m_{i}^{2}(h)+\delta m_{i}^{2}(h, T)\right)\right. \\
\left.+\frac{\partial^{2} V_{\mathrm{th}}^{i}}{\partial \phi_{j}^{2}}\left(m_{i}^{2}(h)+\delta m_{i}^{2}(h, T), T\right)\right]
\end{array}
$$

where $\phi_{j}=h, G_{0}, S_{0}$. Since we only consider excursions along the $h$ direction, there are no mixed mass terms, and mass corrections for all singlets and Goldstones respectively are equal. ${ }^{5}$

Note that while we only numerically solve for the mass corrections of the scalars, these mass corrections will include contributions due to gauge bosons and fermions, which decouple correctly away from the high-temperature limit. We will address some subtleties related to finding consistent numerical solutions to these gap equations, and the effect of derivatives of $\delta m_{\phi_{j}}^{2}$, in the next section.

In defining the effective potential Eq. (3.17), we are essentially using only the one-loop potential as in Eq. (3.5). This represents a great simplification, since calculation of the twoloop lollipop in full generality and at finite temperature [82] may be very onerous in a general BSM theory. Furthermore, implementing the factor-of- $2 / 3$ "fix" to correctly count sunset contributions may be nontrivial at finite-temperature. Fortunately, we can show that omitting both of these contributions is justified for our cases of interest.

First, the lollipop is suppressed relative to the dominant one-loop resummed potential by factors of $N$ (where $N$ is related to $N_{S}$ but also the number of Goldstones in the SM) and $\beta<1$. Even so, it represents our dominant neglected contribution. To explicitly check that it is small, it is sufficient to evaluate the dominant lollipop contributions to the $h$ and $S_{0}$ thermal masses in the high-temperature limit. Adapting the loop integral in the high- $T$ limit from [83], this gives

$$
\begin{aligned}
\delta m_{h, L L}^{2}(T)= & \frac{T^{2}}{16 \pi^{2}}\left[6 \lambda^{2}\left(\log \frac{m_{h}^{2}}{T \mu_{R}}+1.65\right)\right. \\
& \left.+N_{S} \lambda_{H S}^{2}\left(\log \frac{\left(m_{h}+2 m_{S}\right)^{2}}{9 T \mu_{R}}+1.65\right)\right]
\end{aligned}
$$

\footnotetext{
$\overline{5}$ We can also evaluate the potential along the $S_{0}$ axis, in which case the mass corrections for $S_{0}$ and $S_{k}$ with $k \geq 1$ have to be treated separately. Note that Eq. (3.19) is also a function of the gauge boson masses and thermal masses, which are set by Eq. (3.18), as well as the fermion masses.
}

$$
\begin{aligned}
\delta m_{S, L L}^{2}(T)= & \frac{T^{2}}{16 \pi^{2}}\left[\left(N_{S}+2\right) \lambda_{S}^{2}\left(\log \frac{m_{S}^{2}}{T \mu_{R}}+1.65\right)\right. \\
& \left.+4 \lambda_{H S}^{2}\left(\log \frac{\left(m_{S}+2 m_{h}\right)^{2}}{9 T \mu_{R}}+1.65\right)\right]
\end{aligned}
$$

These diagrams are evaluated with improved propagators. In order for the calculation to be reliable, the ratios of lollipop to resummed one-loop mass corrections must satisfy

$$
\begin{aligned}
r_{L L}^{k} \equiv & \frac{1}{\delta m_{k}^{2}\left(h=0, T_{c}\right)} \\
& \times\left.\delta m_{k, L L}^{2}\left(T_{c}\right)\right|_{m_{h} \rightarrow \sqrt{m_{h}^{2}+\delta m_{h}^{2}}, m_{S} \rightarrow \sqrt{m_{S}^{2}+\delta m_{S}^{2}}} \lesssim 0.1,
\end{aligned}
$$

for $k=h, S$ when the high- $T$ approximation is valid at the origin. (As explained in Sect. 2.3, in all operations involving the effective potential or its derivatives, we always only use the real part.)

Second, the sunset contribution is suppressed relative to the dominant one-loop-resummed potential by factors of $h / T, N^{2}$ and $\beta<1$. More importantly, we expect the most important improvement of our partial dressing computation, compared to the standard truncated full dressing computation, to be the correct inclusion of finite-temperature effects, and the associated decoupling of heavy modes from the plasma away from the origin in field space. This decoupling will dominantly be due to the increased mass of the singlets as $h$ evolves away from the origin, rather than from the two-loop sunset contributions. Since the former is correctly captured by using finite-temperature gap equations and effective potential, the effect of sunsets should be small compared to the lollipop.

Third, we should also ensure that the equivalent of $\beta^{3}$ in our theory is sufficiently small at $T=T_{c}$. This is very straightforward using Eq. (3.11), where $\delta m_{1}$ is the unimproved Higgs or Singlet one-loop thermal mass (using $\mathrm{m}^{2}$ in propagators), and $\delta m_{D}^{2}$ is well approximated by the difference between the improved (using $M^{2}$ ) and un-improved thermal mass. Explicitly, we define two parameters:

$\beta_{k} \equiv \frac{\left|\delta m_{k}^{2}\left(0, T_{c}\right)-\delta m_{k}^{2}\left(0, T_{c}\right)\right|_{\delta m_{i}^{2} \rightarrow 0} \mid}{\left.\delta m_{k}^{2}\left(0, T_{c}\right)\right|_{\delta m_{i}^{2} \rightarrow 0}}$

for $k=h, S . \beta_{h}^{3}$ and $\beta_{S}^{3}$ then give the size of the error terms from the most important neglected three-loop diagrams and should be less than $\sim 0.1$.

Finally, two-loop zero-temperature corrections are small if $N \lambda \ll 4 \pi$, which simply restricts the weakly coupled parameter space we can explore. Our PD computation of the phase transition strength $v_{c} / T_{c}$ is then robust when all $r_{L L}^{h, S}$ and $\beta_{h, S}^{3}$ are small. 


\subsection{Future directions}

There are several conceptually straightforward ways to extend the procedure outlined in Sect. 3.2:

1. Even though it cannot be done by simple construction and manipulation of the one-loop effective potential, it would be straightforward to construct the gap equations for the gauge bosons [85] and solve them together with the gap equations for the scalars.

2. With a general finite-temperature expression for the 2loop lollipop shown in Fig. 2, we could evaluate its derivative, substitute $m^{2} \rightarrow m^{2}+\delta m^{2}$ and by adding it to Eq. (3.17) [but not Eq. (3.19)] correctly include all lollipop contributions, as in Eq. (3.8)

3. In regimes where the gap equation Eq. (3.19) can be approximated by a high-temperature expansion, one could implement the $2 / 3$ factor fix of Eq. (3.4) to correctly count sunset graphs at 2- and higher loop order. It is unclear how to implement this fix in the full finitetemperature gap equation, but as we discuss when we introduce optimized partial dressing (OPD) in Sect. 4.2.3, the high-temperature expansion of the gap equation (but not the potential) is sufficient in most BSM calculations.

4. We have applied partial dressing without explicitly checking that fermion and gauge boson effects are correctly accounted for at 2- and higher-loop order. We leave this investigation to future work, but since the dominant BSM effects on the Higgs potential in our models of interest come from the scalar sector, we expect our procedure to be valid.

Note that we explicitly check in our calculation whether the above-mentioned extensions (2) and (3) are numerically significant.

There is also a more involved question, which is how to extend partial dressing for field excursions along several field directions at once, when those field directions cannot be related by a symmetry. The partial dressing procedure unambiguously defines the potential along any one field direction, as long as all other fields are at the origin. In an example with two fields, let us denote as $V_{\text {eff }}^{\mathrm{pd}_{\mathrm{i}}}\left(\phi_{1}, \phi_{2}\right)$ the effective resummed one-loop potential obtained by integrating $\partial V_{\text {eff }} / \partial \phi_{i}$. It is straightforward to show that $V_{\text {eff }}^{\mathrm{pd}_{1}}\left(\phi_{1}, \phi_{2}\right) \neq$ $V_{\mathrm{eff}}^{\mathrm{pd}_{2}}\left(\phi_{1}, \phi_{2}\right)$, with the difference being of super-daisy order $\mathcal{O}\left(\beta^{2}\right)$. This may not be numerically significant in a given case, but it would be of interest to extend the partial dressing procedure to consistently define

$V_{\mathrm{eff}}^{\mathrm{pd}}\left(\phi_{1}, \phi_{2}\right)$.

Since the above can be evaluated unambigiously diagrammatically, and since the partial dressing procedure was vali- dated in [87] by comparing this to the substitution of $M^{2}$ into the first derivative of the potential, there presumably exists a way of generalizing this substitution procedure to obtain a general potential as a function of multiple fields. This is of particular relevance to BSM models where the Higgs mixes with BSM scalars [24,34,37], which constitute an important class of models that can give a strong electroweak phase transition.

\subsection{Comparison with dimensional reduction}

While the majority of the BSM community uses the methods of perturbative resummation, there are a number of demonstrably incorrect methods, e.g. the $V_{\text {ring }}^{i}\left(m_{i}^{2}(h, S), T\right)$ potential, which we have addressed thus far. However, there is another school of thought more associated with nonperturbative methods that typically employs dimensional reduction to address finite- $T$ field theories. This method was first proposed in [106] as way to isolate the high- $T$ behavior. This is quite natural in examining the high- $T$ limit of the theory when the radius of the thermal circle is very small and one can reformulate the theory as a three-dimensional effective theory with $T$ as the expansion parameter. (Depending on the theory in question there can be multiple $T$-dependent scales involving different coupling constants or factors of $\pi$.) The resulting $3 \mathrm{D}$ theory was implemented on the lattice in [107] where a number of possible benefits were explored. In particular, for finite $T$ gauge theories, the value of the nonperturbative magnetic mass term can imply a reduced radius of convergence for perturbation theory, necessitating the use of lattice methods. By using an effective 3D description, depending on the theory in question, non-perturbative and perturbative physics can be separated and a simpler 3D lattice simulation can be used rather than a 4D version $[107,108]$.

Dimensional reduction applied to finite- $T$ has also been championed for its potential universality of the 3D effective theory $[23,109]$. This emphasis arises because at high $T$ and near the SM EW transition the relevant modes reduce to a solely super-renormalizable 3D EFT of scalars and gauge field modes. However, the hoped-for universality breaks down when investigating phenomena like P-violation where higher dimension operators are needed to accurately describe the physics [110]. Additionally, it is important to remember that in any EFT a matching calculation must be done, and the desired accuracy depends in principle on both the matching and the number of higher-dimensional operators that are included [110]. More generally, in BSM physics there can be many additional fields with masses that arise from sources other than EWSB while also coupling to the Higgs. Therefore to accurately model a phase transition one may have to keep track of these additional fields. From the EFT perspective their contributions must either be included directly as soft modes, or there might be a small radius of conver- 
gence of the EFT with many higher dimensional operators needed. In either of these cases the EFT description immediately becomes a less universal prediction, and more lattice studies would have to be carried out for each of these theories. Otherwise, the effects predicted from the thermal EFTs are no more accurate than the $4 \mathrm{D}$ theory they are perturbatively matched to in the first place. Additionally, on top of the original perturbative calculation required for matching, there could be multiple layers of EFTs needed to correctly describe the physics at high $T$ if there are multiple scales involved.

While dimensional reduction is a very useful tool for finite- $T$ QFT, especially for physics driven by gauge theories, it is not always the most efficient tool for BSM theories. In the BSM context, the lack of universality or multiple EFTs required combined with the necessity of matching with resummation shows that there is a great deal of utility in the perturbative resummation techniques we discuss here. This is especially true in the context of new scalar fields with $m \sim T_{E W}$ which is one of the most intriguing phenomenological possibilities that can be studied in current or future collider experiments. Additionally, as we show in later sections, the fast numeric techniques we propose demonstrate the efficiency of using this method for BSM physics and renders this approach at least as useful as dimensional reduction until lattice methods become more numerically efficient. While ultimately dimensional reduction and lattice methods would be ideal if widely available and carefully matched to each individual BSM theory, our methods provide a very useful way to explore the most promising BSM directions for future theoretical and experimental exploration, in particular for new scalar sectors beyond the Higgs.

\section{Computing the strength of the phase transition}

We now apply the procedure outlined in Sect. 3.2 the SM $+N_{S} \times S$ BSM benchmark model with unbroken $O\left(N_{S}\right)$ symmetry in the $T=0$ ground state.

\subsection{Zero-temperature calculation}

The tree-level scalar potential is given by Eqs. (2.2) and (2.3). All field excursions in the region of parameter space we study (no Higgs-Singlet mixing in the ground state) can be considered without loss of generality to be in either the $h$ or $S_{0}$ direction.

For each choice of $N_{S}$, there are three Lagrangian BSM parameters, $\mu_{S}, \lambda_{H S}$ and $\lambda_{S}$. We match these to three physical input parameters which are computed at one-loop level in the $\overline{\mathrm{MS}}$ scheme (in addition to matching the SM Higgs potential parameters $\lambda, \mu$ to $m_{h}$ and $v$ ): (a) The mass of the singlet in our vacuum

$$
m_{S}^{2}=m_{S}^{2}(v)=\left.\frac{\partial^{2} V_{\mathrm{eff}}^{T=0}}{\partial S_{0}^{2}}\right|_{h=v}=\mu_{S}^{2}+\lambda_{H S} v^{2}+\cdots
$$

(b) The singlet-Higgs cubic coupling

$$
\lambda_{h S S}^{\text {loop }}=\lambda_{h S S}^{\text {loop }}(v)=\left.\frac{1}{2} \frac{\partial^{3} V_{\text {eff }}^{T=0}}{\partial S_{0}^{2} \partial h}\right|_{h=v}=\lambda_{H S} v+\cdots
$$

(c) The singlet quartic coupling

$$
\lambda_{S}^{\text {loop }}=\lambda_{S}^{\text {loop }}(v)=\left.\frac{1}{6} \frac{\partial^{4} V_{\mathrm{eff}}^{T=0}}{\partial S_{0}^{4}}\right|_{h=v}=\lambda_{S}+\cdots
$$

The renormalization scale $\mu_{R}$ is set to $m_{S}(v)$. In all calculations, we vary this scale choice up and down by a factor of 2 in order to estimate the uncertainty due to higher-order zero-temperature corrections. For a given set of input parameters, we compute whether the singlet is stable at the origin when $h=0$, and if not, the location of the local minimum $\left(h, S_{0}\right)=(0, w)$. When the singlet is unstable at the origin, we impose the vacuum stability condition

$V_{\mathrm{eff}}^{T=0}(v, 0)<V_{\mathrm{eff}}^{T=0}(0, w)$

to ensure the EWSB vacuum is the preferred one for our universe.

The collider phenomenology of the $N_{S}=1$ case, with TFD resummation and in the on-shell renormalization scheme, was studied previously in [111]. The following three collider observables are of interest to probe this scenario: ${ }^{6}$

- A measurement of the triple-Higgs coupling with 5\% precision at $1 \sigma$, which is more pessimistic than recent estimates of the achievable precision [97].

- A search in the VBF jets + MET channel for direct singlet pair production via $h^{*} \rightarrow S S$.

- A measurement of the $Z h$ production cross section deviation from the SM with a $1 \sigma$ precision of $0.3 \%$ at a TLEPlike lepton collider.

Together, it was found that these measurements provide coverage of the entire parameter space where a strong phase transition is possible.

We analyze the same model as the authors of [111], generalized to $N_{S} \geq 1$, while comparing standard truncated full

\footnotetext{
${ }^{6}$ We assume $3 \mathrm{ab}^{-1}$ of luminosity at $100 \mathrm{TeV}$ throughout.
} 
dressing to our new partial dressing procedure. We therefore compute the same observables:

(a) The Higgs cubic coupling

$$
\lambda_{h h h}^{\text {loop }}=\lambda_{h h h}^{\text {loop }}(v)=\left.\frac{1}{6} \frac{\partial^{3} V_{\mathrm{eff}}^{T=0}}{\partial h^{3}}\right|_{h=v}=\frac{m_{h}^{2}}{2 v}+\cdots
$$

To obtain the fractional deviation, we compare this to the value of $\lambda_{h h h}^{\text {loop }}$ computed without the $S$ contribution, rematched to the SM parameters with the same choice of renormalization scale as the calculation with the singlet.

(b) Singlet pair production cross section at $100 \mathrm{TeV}$ colliders: obtained in MadGraph 5 [123] by using $\lambda_{h S S}^{\text {loop }}$ as the singlet-Higgs tree-level coupling, scaled up by a factor of $N_{S}$.

(c) The fractional $Z h$ cross section shift at lepton colliders can be computed using the results in $[124,125]$ :

$\delta \sigma_{Z h}=\frac{N_{S}}{2} \frac{\left|\lambda_{H S}\right|^{2} v^{2}}{4 \pi^{2} m_{h}^{2}}\left[1+F\left(\tau_{\phi}\right)\right]$

with the replacement $\lambda_{H S} v \rightarrow \lambda_{h S S}^{\text {loop }}$. The loop function $F(\tau)$, with $\tau_{\phi}=m_{h}^{2} / 4 m_{S}^{2}$, is given by

$$
F(\tau)=\frac{1}{4 \sqrt{\tau(\tau-1)}} \log \left(\frac{1-2 \tau-2 \sqrt{(\tau(\tau-1))}}{1-2 \tau+2 \sqrt{(\tau(\tau-1))}}\right) .
$$

Note that we estimate changes in cross-sections from the changes in potential couplings. This ignores finitemomentum contributions of new particles in loops to the cross section, but for $m_{S} \gtrsim m_{h}$, this is a good approximation.

Note that for these zero-temperature calculations, we do not resum mass corrections in the partial dressing scheme. The procedures of Sect. 3.2 can easily be applied to the zero-temperature potential as well, but we have extensively checked that they only have insignificant effects on the matched parameters and corresponding observables. This is expected, since mass resummation at finite temperature is required due to IR-divergent effects which are absent at zero temperature. $^{7}$

\subsection{Finite-temperature calculation}

We are interested in finding regions of parameter space where the phase transition is strongly one-step $\left(v_{c} / T_{c}>0.6\right)$ or two-step.

\footnotetext{
7 Note, however, that in the finite-temperature calculation, the zerotemperature potential gives important contributions beyond $T^{2}$ order.
}

For each set of input parameters we match the potential, compute zero-temperature observables, and then compute the finite-temperature potential at different temperatures until we find $T=T_{c}$ where the local minimum at the origin and a local minimum at $v=v_{c}$ are degenerate. The finite-temperature potential is given by Eq. (3.17), where the mass corrections (or thermal masses) $\delta m_{i}^{2}$ are computed differently depending on the resummation scheme. If the singlet is unstable at the origin at $T=0$, we also compute the minimum temperature $T_{S}$ where thermal effects stabilize the singlet. If $T_{S}>T_{c}$, the transition is two-step and we do not analyze it further. In order for our calculation to be reliable, $r_{L L}^{h, S}$ and $\beta_{h, S}$ as defined in Eqs. (3.21) and (3.22), have to be small.

We compare the three different schemes: truncated full dressing (TFD), partial dressing (PD) and optimized partial dressing (OPD). Some of the numerical calculations are performed in Mathematica, and some in a custom-built $\mathrm{C}++$ framework. For numerical evaluation, $\mathrm{c}++$ is operation-byoperation $\sim 1000 \times$ faster than Mathematica, but the latter is much more versatile and often preferred for exploring different BSM scenarios. Whenever evaluating the full thermal potential $V_{\text {th }}$ or its derivatives without approximations, we use pre-computed lookup tables.

The TFD calculation, which is the standard method for BSM calculations used in [111] and many other analyses, is simple enough that $v_{c} / T_{c}$ can be found for a given parameter point using about $\sim \mathcal{O}(1$ minute $)$ of CPU time in Mathematica. On the other hand, the complete implementation of PD is so numerically intensive that only the $\mathrm{C}++$ code can be realistically used, and evaluation times for a single parameter point range from 10 seconds to many minutes, indicating that PD is of order $10^{4}$ times more numerically intensive than TFD. Fortunately, a series of carefully chosen approximations allows partial dressing to be implemented in Mathematica, with only $\mathcal{O}(10 \%)$ higher CPU cost than TFD, but identical results as PD. This is the OPD scheme, and we hope its ease of implementation and evaluation will be useful for future BSM analyses. We now briefly summarize and contrast the salient features of each resummation implementation.

\subsubsection{Truncated full dressing (TFD)}

This is just the standard thermal mass resummation, using $\delta m_{i}^{2}=\Pi_{i} \propto T^{2}$, see Eq. (2.22). This thermal mass is substituted into the first derivative of the potential, since there are no differences between full and partial dressing when substituting only the $T^{2}$ piece of the thermal mass. The thermal potential derivative $V_{\text {th }}^{\prime}$ is evaluated numerically without any approximations.

The dominant errors in the perturbative expansion arise from neglecting the two-loop lollipop, and miscounting the two-loop daisy. We define the corresponding error term 
$\Delta^{\mathrm{TFD}} \equiv \max \left(r_{L L}^{h}, r_{L L}^{S}, \beta_{h}, \beta_{S}\right)$

to perform the perfunctory check that the calculation is reliable. However, is important to note that $\Delta^{\text {TFD }}$ will greatly underestimate the error of the TFD calculation, since it does not include the $\mathcal{O}(m / T)$ errors in the truncated thermal mass. ${ }^{8}$ It is precisely this error that will be explored by comparing the TFD calculation to (O)PD calculation.

\subsubsection{Partial dressing $(P D)$}

This implements the scheme outlined in Sect. 3.2 verbatim. For each temperature $T$ of interest, the algebraic gap equation Eq. (3.19) is constructed by substituting $m_{i}^{2} \rightarrow m_{i}^{2}+\delta m_{i}^{2}$ into the second derivative of the full finite-temperature thermal potential $V_{\text {th }}^{\prime \prime}$ and $V_{\mathrm{CW}}^{\prime \prime}$. Solutions $\left\{\delta m_{i}^{2}(h)\right\}$ are obtained numerically for each value of $h$. The resulting solutions $\delta m_{i}^{2}(h, T)$ are substituted into the first derivative of the full effective potential Eq. (3.17), again without any approximations. The dominant errors arise from neglected lollipops and three-loop diagrams, and can be estimated with the error term

$\Delta^{\mathrm{PD}} \equiv \max \left(r_{L L}^{h}, r_{L L}^{S}, \beta_{h}^{3}, \beta_{S}^{3}\right)$

In practice, considerable complications arise when attempting to solve the gap equation Eq. (3.19). We find the best possible solution (whether an exact solution exists or not) by minimizing $|\operatorname{Re}(\mathrm{LHS})-\operatorname{Re}(\mathrm{RHS})|$ with respect to different choices of $\left\{\delta m_{i}^{2}\right\}$. A unique solution to the gap equations can always be found at the origin of field space $(h, S)=(0,0)$. However, sometimes no exact solution $\left\{\delta m_{i}^{2}\right\}$ can be found. This occurs for values of $h$ where some scalars become tachyonic, which is for example the case across the energy barrier. In that case, we use the closest approximate solution, or discard the solution and interpolate across these pathological values of $h$. Both methods give very similar results, and in plots we use the former. The approximate solution usually still satisfies the gap equation at the $\sim 1$ to $10 \%$ level, which on the face of it appears sufficient for a one-loop exact quantity.

Far more troubling is that for all other non-zero $h$-values, there exists not one but many numerical solutions to the algebraic gap equation. Apart from the computational intensity of $\mathrm{PD}$, this is one of the confusing aspects which prompted us to develop OPD. ${ }^{9}$ Physically, one would expect the solution of the gap equation to correspond to the limit of an iteration,

\footnotetext{
${ }^{8}$ In particular, the $m / T$ errors are expected to dominate the $\beta$-size errors, since the differences between full and partial dressing, which are order $\beta$, only show up at subleading order in $T$.

${ }^{9}$ We have checked that our choice of solving a set of coupled gap equations for the scalar thermal masses, while using the analytical approximations for the gauge boson thermal masses Eq. (2.22), is not responsible for either the absence or abundance of gap equation solutions at different values of $h$.
}

whereby propagators in diagrams contributing to the effective potential are recursively dressed with additional one-loop bubbles until their second derivative with respect to the field is consistent with the mass used in the propagators. Therefore, one way we attempt to "find" our way towards the physically relevant gap equation solution is by iteration, where the $n+1$ step is given by

$$
\begin{aligned}
\delta m_{\phi_{j}}^{2}(h, T)_{n+1}= & \sum_{i}\left[\frac{\partial^{2} V_{\mathrm{CW}}^{i}}{\partial \phi_{j}^{2}}\left(m_{i}^{2}(h)+\delta m_{i}^{2}(h, T)_{n}\right)\right. \\
& \left.+\frac{\partial^{2} V_{\mathrm{th}}^{i}}{\partial \phi_{j}^{2}}\left(m_{i}^{2}(h)+\delta m_{i}^{2}(h, T)_{n}, T\right)\right]
\end{aligned}
$$

for fixed $h, T$. The iteration starts at $\delta m_{\phi_{j}}^{2}=0$ and continues until the result converges. However, this series does not always converge, instead oscillating between two or more values. This is always the case when there is no exact solution to the gap equation, but can also occur when there are one or more solutions. We numerically circumvent this issue by requiring the solution $\delta m_{i}^{2}(h, T)$ to be a set of smooth functions. Therefore, once solutions $\delta m_{i}^{2}(h, T)$ are obtained for a grid of $h$-values and fixed $T$, we use smoothing to eliminate numerical artifacts (or the jumps due to imperfect or multiple solutions), and interpolate across regions without solutions to obtain a smooth set of functions describing the thermal masses.

\subsubsection{Optimized partial dressing (OPD)}

As we will discuss in Sect. 4.3, the results obtained via PD seem physically reasonable. However, it has two major disadvantages. First, its computational complexity and intensity would likely hamper adoption for other BSM calculations. Second, some of the numerical tricks used to obtain reasonable solutions to the gap equation are slightly unsatisfying. We are therefore motivated to develop the more streamlined OPD resummation scheme that is numerically efficient and does not suffer from either the absence of gap equation solutions for some ranges of $h$ values, nor the preponderance of solutions for all other nonzero $h$ values. Encouragingly, the physical results of this OPD procedure are practically identical to PD.

The first important observation, which is very well-known, is that the high-temperature approximation for the thermal potential, Eq. (2.13), has the expected error terms of order $(m / T)^{n+1}$ if truncated at order $(m / T)^{n}$ (for $n \leq 2$ ), but is accurate for masses as large as $m \sim(1-3) \times T$ if the log terms are included. This applies both to $V_{\text {th }}$ and its derivatives.

Given that the low-temperature approximation Eq. (2.14) can easily be expanded to high enough order to be accurate for $m \sim T$, this implies that a piece-wise approximation 
for the thermal functions $J_{B, F}$ can be used in Eq. (3.17) to evaluate the effective potential, as well as its derivatives:

$$
\begin{aligned}
& J_{B}^{\text {piece-wise }}\left(y^{2}\right)= \begin{cases}J_{B}^{\mathrm{high}-T}\left(y^{2}\right) & \text { for } y^{2} \leq 1.22 \\
\tilde{J}_{B}^{(3)}\left(y^{2}\right) & \text { for } y^{2}>1.22\end{cases} \\
& J_{F}^{\text {piece-wise }}\left(y^{2}\right)= \begin{cases}J_{F}^{\mathrm{high}-T}\left(y^{2}\right) & \text { for } y^{2} \leq 1.29 \\
\tilde{J}_{F}^{(2)}\left(y^{2}\right) & \text { for } y^{2}>1.29 .\end{cases}
\end{aligned}
$$

This gives percent-level or better accuracy for $J_{B}$ and its first two derivatives and $J_{F}$ and its first derivative, for all positive $y^{2}$. For negative $y^{2}$ (corresponding to tachyonic masses) the accuracy is $\sim 10 \%$ for $m^{2}=-10 T^{2}$, but such negative $\mathrm{m}^{2}$ are rarely encountered after thermal mass corrections are added. Evaluation of this piece-wise approximate form of $J_{B, F}$ is very fast in Mathematica.

This definition of the thermal effective potential $V_{\text {th }}$ also allows the algebraic gap equation Eq. (3.19) to be defined entirely analytically (as opposed to numerically), even if the ultimate solutions have to be found numerically. This represents a huge simplification and allows Mathematica to find solutions much easier than for the full $V_{\text {th }}$ defined via lookuptables. In its full piece-wise defined form, the thermal functions of Eq. (4.11) still allow for the study of e.g. decoupling effects as particles become heavy, which will be important in the future study of EFTs at finite temperature.

However, for the study of strong phase transitions induced by BSM thermal effects, we can make another important simplification. Thermal mass resummation is only needed to obtain accurate results when $m \lesssim T$. Therefore, we are justified in constructing the gap equation Eq. (3.19) using only the high-temperature approximation for the thermal potential (and the usual $V_{\mathrm{CW}}$ ). The resulting solutions for the mass corrections $\left\{\delta m_{i}^{2}(h, T)\right\}$ are practically identical to the solutions obtained with the full finite-temperature potential, except for some modest deviations in regions where $m \gtrsim T$. Even so, the resulting effective potential obtained by integrating Eq. (3.17) is practically identical in those regions as well, since thermal effects of the corresponding degrees of freedom are no longer important. Using only $J_{B, F}^{\text {high }-T}$ in the gap equation makes finding solutions so fast that the associated computational cost becomes a subdominant part of the total CPU time required for finding $v_{c} / T_{c}$, making this OPD method only $\mathcal{O}(10 \%)$ slower than the standard TFD method (even when the TFD method uses the same piecewise defined thermal functions in the effective potential).

\section{Alternative formulation of the gap equations}

The absence of solutions to the algebraic gap equation Eq. (3.19) encountered in PD indicate an overconstrained system, meaning there might be missing variables we should also solve for. Furthermore, the numerical tricks utilized in the PD implementation to obtain $\delta m_{i}^{2}(h, T)$ solutions for a given $T$ were justified by appealing to the required continu- ity of the solution (interpolation, smoothing) and the physical interpretation of the gap equation (selecting the correct solution by guiding the numerical root-finding procedure with iteration).

All of these considerations point towards a slightly modified form of the gap equation which appears more consistent with the partial dressing procedure. Recall that the gap equation for both the full and partial dressing procedures, Eq. (3.4), was originally defined by substituting $m^{2} \rightarrow M^{2}$ in $V^{\prime \prime}$ of the $\phi^{4}$ theory:

$M^{2}=m^{2}+\left.V_{1}^{\prime \prime}\right|_{m^{2} \rightarrow M^{2}}$.

This yields the gap equation used in the PD procedure Eq. (3.19), which is an algebraic equation that is solved for $\left\{\delta m_{i}^{2}\right\}$, a priori separately for each $(h, T)$. Alternatively, one could define the gap equation by taking the effective potential of partial dressing $V_{1}^{\prime p d}=\left.V_{1}^{\prime}\right|_{m^{2} \rightarrow M^{2}}$ and differentiating it once more with respect to the field:

$M^{2}=m^{2}+\left[\left.V_{1}^{\prime}\right|_{m^{2} \rightarrow M^{2}}\right]^{\prime}$.

In the context of our BSM benchmark model, the corresponding gap equations are

$$
\begin{aligned}
\delta m_{\phi_{j}}^{2}(h, T)= & \sum_{i} \frac{\partial}{\partial \phi_{j}}\left[\frac{\partial V_{\mathrm{CW}}^{i}}{\partial \phi_{j}}\left(m_{i}^{2}(h)+\delta m_{i}^{2}(h, T)\right)\right. \\
& \left.+\frac{\partial V_{\mathrm{th}}^{i}}{\partial \phi_{j}}\left(m_{i}^{2}(h)+\delta m_{i}^{2}(h, T), T\right)\right]
\end{aligned}
$$

At each point in $(h, T)$ space, these gap equations are algebraic relations of $\left\{\delta m_{i}^{2}\right\}$ as well as the derivatives at that point $\left\{\frac{\partial \delta m_{i}^{2}}{\partial \phi_{j}}\right\}$. The gap equations are now partial differential equations: The additional variables (the derivatives of $\delta m_{i}^{2}$ ) guarantee that solutions exist at every point, ${ }^{10}$ while the continuity condition of the PDEs

$\delta m_{i}^{2}(h+\Delta h, T)=\delta m_{i}^{2}(h, T)+\Delta h \frac{\delta m_{i}^{2}}{\partial h}(h, T)+\mathcal{O}\left(\Delta h^{2}\right)$

restricts the number of numerical solutions, selecting the unique physical solution. This eliminates both numerical problems of the original PD procedure.

In practice, constructing Eq. (4.14) is very challenging in the full finite-temperature formulation of $V_{\mathrm{th}}$, since it involves second derivatives of the thermal functions which are very computationally costly to evaluate. However, in the high-

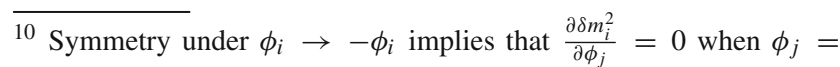
0 . Since we only consider excursions along the $h$-direction, only the derivatives with respect to $h$ are ever nonzero. At the origin both kinds of gap equations are the same, but at that point the algebraic gap equation always has a unique solution anyway.
} 
temperature approximation ${ }^{11}$ Eq. (4.14) becomes a very simple analytical set of equations that Mathematica can easily solve numerically for $\left\{\delta m_{i}^{2}\right\}$ after eliminating the derivatives $\left\{\frac{\partial \delta m_{i}^{2}}{\partial h}\right\}$ via the continuity condition Eq. (4.15), making the solution at $(h+\Delta h, T)$ dependent on the solution obtained at $(h, T)$. The resulting $(h, T)$-dependent mass corrections $\delta m_{i}^{2}(h, T)$ are well-behaved, defined everywhere, and unique almost everywhere. ${ }^{12}$

In summary, the OPD method uses the piece-wise defined thermal functions Eq. (4.11) in the effective potential Eq. (3.17), and the high-temperature approximation in the gap equations Eq. (4.14). The gap equations are PDEs instead of simple algebraic relations, which leads to solutions for the mass corrections which are continuous and well-defined for all $(h, T)$ in the regions of interest.

Using gap equation Eq. (4.13) instead of Eq. (4.12) amounts to treating resummation identically in the effective potential and the gap equation derived from that potential. While this seems reasonable, the important question is whether this particular algebraic procedure of constructing a gap equation by manipulating the one-loop potential expressions, and inserting the resulting mass solution back into the potential, is equivalent (up to some order) to the diagrammatic procedure of computing various higher-order contributions to the effective potential. As we reviewed in Sect. 3, the authors of [87] showed that using the original gap equation Eq. (4.12), this equivalence was accurate up to differences of $\mathcal{O}\left(\beta^{3}\right)$, the neglected two-loop lollipop, and the miscounted two-loop sunset, see Fig. 2 . We have checked that using the new gap equation Eq. (4.13) is equivalent to the same order, the only difference being the precise nature of how the twoloop sunset is miscounted. This means that the $2 / 3$ correction factor in Eq. (3.8) is modified at subleading (i.e. 3-loop sunset) order.

In Sect. 3.2 we argued that the sunset error is subdominant to the $\mathcal{O}\left(\beta^{3}\right)$ and lollipop errors, which we check explicitly are small. We therefore expect the numerical difference between $\delta m_{i}^{2}(h, T)$ solutions obtained in the OPD and PD scheme to be even smaller, and as we show below, this is indeed the case. That being said, since the gap equation in the OPD scheme is explicitly written in the high-temperature approximation, inserting the $2 / 3$ correction factor would be very straightforward.

Finally, one might also worry that the piece-wise defined thermal potential, or the high-temperature approximate gap equation, would be of insufficient accuracy in regions where large cancellations are important, or where masses are very

\footnotetext{
11 One could also use the piece-wise definition of the thermal functions Eq. (4.11), but obtaining a solution takes $\mathcal{O}(10)$ times longer. This can still be useful for studying decoupling effects and matching to EFTs.

12 In some regions there are multiple near-degenerate solutions, but the ambiguity is not physically significant.
}

tachyonic and the piecewise approximation is not very accurate. However, thermal resummation prevents masses squared inserted in the thermal potential from being too negative, and in regions where the transition between the highand low-temperature approximation occurs, the exact solution to the gap equation becomes less numerically relevant since the calculation reduces to a fixed-order one.

\subsection{Comparing resummation schemes}

Here we illustrate the differences between the three resummation schemes for an example point in the parameter space of the $\mathrm{SM}+N_{S} \times S$ model. The differences, in particular of the evaluated thermal mass corrections at $T=T_{c}$, will inform our discussion of the different physical predictions generated by the new (O)PD vs the standard TFD scheme in the next section. It will also demonstrate that PD and OPD are nearly equivalent.

We focus on regions of parameter space where both TFD and (O)PD produce a sizable first-order one-step phase transition. This allows us to show the resulting Higgs potentials at $T=T_{c}$ side-by-side, but by necessity restricts our attention to regions of parameter space where all calculation schemes give similar physical predictions. Even so, the differences are very clear in detail and allow us to understand the regions of parameter space where the physical differences are more significant.

Figure 3 shows the effective Higgs potential and mass corrections for the parameter point $\left(N_{S}, m_{S}, \lambda_{h S S}^{\text {loop }} / v, \lambda_{S}^{\text {loop }}\right)=$ $(3,300 \mathrm{GeV}, 1.52,0.5)$. Immediately we see that PD and OPD give nearly identical functional forms of the mass corrections $\delta m_{h, G, S}^{2}\left(h, T_{c}\right)$ once OPD solutions are interpolated. As a result, the effective potential and obtained values of $v_{c}$ and $T_{c}$ are also nearly identical. We have checked that this holds true across the parameter space. Therefore, the numerically extremely efficient OPD method can be used in place of the numerically costly PD calculation. ${ }^{13}$

The mass corrections obtained with (O)PD behave in a physically reasonable manner, being maximal near the origin and generally decreasing as $h$ increases and the various degrees of freedom acquire more mass, reducing their participation in the thermal plasma. The physical Higgs and Goldstone mass corrections behave differently away from the origin. Compared to the constant TFD prediction, the (O)PD thermal masses are smaller by $\mathcal{O}(40 \%)$ or more. This is typ-

\footnotetext{
${ }^{13}$ For one-step transitions with very large couplings, where the zerotemperature singlet mass is small near the origin, but significantly larger than the Higgs mass at $h=v$, there are minor $\mathcal{O}(10 \%)$ differences between OPD and PD due to the assumed high- $T$ approximation in the gap equation. However, these differences do not significantly affect the physically important boundaries between different phases of the theory, where the PT is strongly first order, with one-step, or two-step transitions.
} 
----- TFD: $T_{c}=123.9 \mathrm{GeV}, \mathrm{v}_{\mathrm{c}}=130.5 \mathrm{GeV}, \mathrm{v}_{\mathrm{c}} / \mathrm{T}_{\mathrm{c}}=1.05$

PD: $T_{c}=117.4 \mathrm{GeV}, v_{c}=131.8 \mathrm{GeV}, v_{c} / T_{c}=1.12$

OPD: $T_{c}=117.5 \mathrm{GeV}, v_{c}=135.8 \mathrm{GeV}, v_{c} / T_{c}=1.16$

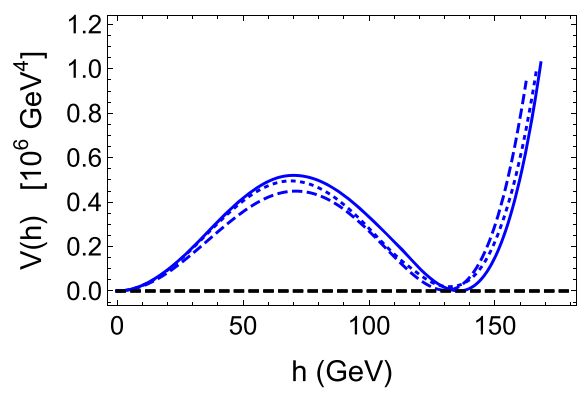

Fig. 3 Effective Higgs Potential (left) and mass corrections $\delta m_{i}^{2}$ (right) for the physical Higgs $(h)$, Goldstones $(G)$, and singlets $(S)$ at $T=T_{c}$ as a function of $h$. Evaluated in TFD, PD and OPD resummation schemes for $N_{S}=3$ and $\left(m_{S}, \lambda_{h S S}^{\text {loop }} / v, \lambda_{S}^{\text {loop }}\right)=(300 \mathrm{GeV}, 1.52,0.5)$. In the right plot, $\delta m_{h}^{2}=\delta m_{G}^{2}$ in the TFD scheme. The dots correspond to $\delta m_{i}^{2}$ in the PD scheme, with gaps indicating regions of the $h$-axis where no exact solution to the gap equation can be found, and the $\delta m_{i}^{2}(h, T)$ functions used to evaluate the potential are obtained by linearly interpo-

ical across the whole parameter space, and explains the most important physical difference between the two schemes. The reduced thermal masses in TFD result in higher temperatures $T_{S}$ where the singlet is stabilized at the origin (if it is unstable at zero temperature). As we show in Sect. 5, this results in larger regions of parameter space where a two-step transition occurs.

\section{Physical consequences}

From a formal point of view, development of the (O)PD thermal resummation scheme is most important in the careful study of thermal decoupling effects, especially when spectra change with field excursions. This is necessary for rigorously understanding Effective Field Theories at finite temperature. We are currently pursuing this line of investigation, and will present the results in a future publication. Additionally, compared to TFD calculations, the new (O)PD formalism makes quantitatively different predictions about the regions of parameter space where a one- or two-step phase transition of sufficient strength for EWBG can occur. This in turn affects the predictions of the EWPT (or other phase transitions in the early universe) for cosmological observations and collider experiments which need to be known reliably for planning such experiments. In more realistic extensions of the SM scalar sector, where the individual masses and couplings are not free parameters as in the $\mathrm{SM}+N_{S} \times S$ benchmark model, this could also affect whether a strong phase transition is possible at all.

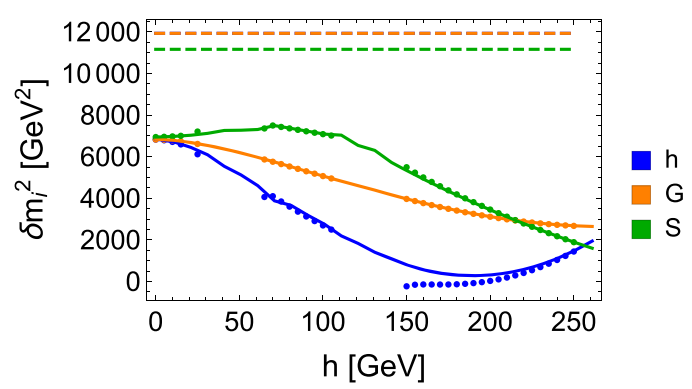

lating between the obtained $\delta m_{i}^{2}$ solutions as a function of $h$. This gives nearly the same $V\left(h, T_{c}\right)$ as OPD. Note that the approximate equality of the three $(\mathrm{O}) \mathrm{PD}$ mass corrections at the origin is a numerical coincidence for this parameter point. Furthermore, the differences in $v_{c}, T_{c}$ between TFD and (O)PD are modest here, but for other choices they can be much more pronounced. This is very important when $T_{c} \sim T_{S}$ and the predicted nature of the transition can change from one-step to two-step, as we discuss in Sect. 5

One way to understand the different predictions of the TFD and (O)PD is to take a slice of parameter space with constant physical singlet mass $m_{S}$ and singlet quartic $\lambda_{S}^{\text {loop }}$ in our EWSB vacuum. The strength of the phase transition, $v_{c} / T_{c}$, is shown along with several other important observables and parameters in Fig. 4 for $N_{S}=6$ and $\left(m_{S}, \lambda_{S}^{\text {loop }}\right)=(150 \mathrm{GeV}, 1.0)$.

We first explain the qualitative features of Fig. 4 which are common to both calculations. For very small Higgs portal coupling $\lambda_{H S} \approx \lambda_{h S S}^{\text {loop }} / v$, the singlet sector has no effect on the EWPT, making it weakly first order or second order as in the SM. The singlet mass, which is $m_{S}^{2}=\mu_{S}^{2}+\lambda_{H S} v^{2}$ at tree-level, is given entirely by the parameter $\mu_{S}^{2}$. As $\lambda_{H S}$ is increased, $\mu_{S}^{2}$ decreases and eventually becomes negative to keep $m_{S}$ fixed. At some point this allows a partial cancellation between $\mu_{S}^{2}$ and $\delta m_{S}^{2}$ along the lines of Eq. (2.25) to occur, resulting in a one-step first order phase transition starting around $\lambda_{h S S}^{\text {loop }} / v \approx 0.55$ in Fig. 4a. This cancellation is only partial, as shown by the plot of total finite-temperature singlet mass at the origin (i). Note from (c) and (d) that $T_{S}$, the minimum temperature at which thermal effects stabilize the singlet at the origin, is lower than $T_{c}$, as required for the singlet to be stable when the Higgs undergoes its one-step phase transition. Increasing the Higgs portal coupling drastically increases $v_{c}$ and hence the strength of the phase transition, see (a) and (b), since it enhances the negative "cubic term" of Eq. (2.25) (from the tree-level Higgs dependence of the singlet mass) while also increasing the singlet thermal mass and therefore enhancing the cancellation of $\mu_{S}^{2}$ and $\delta m_{S}^{2}$, see (i). For $\delta m_{h, S}^{2} \sim \lambda_{H S} T^{2}$, both $T_{c}$ and $T_{S}$ can be schematically 


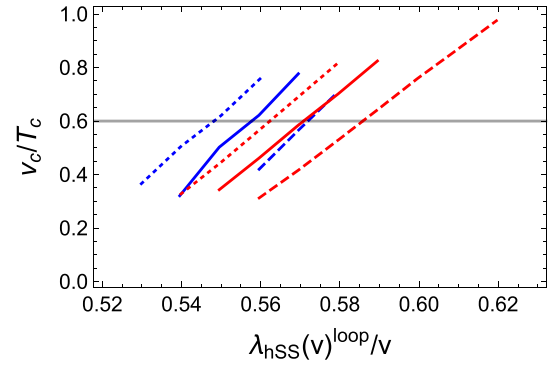

(a) $v_{c} / T_{c}$

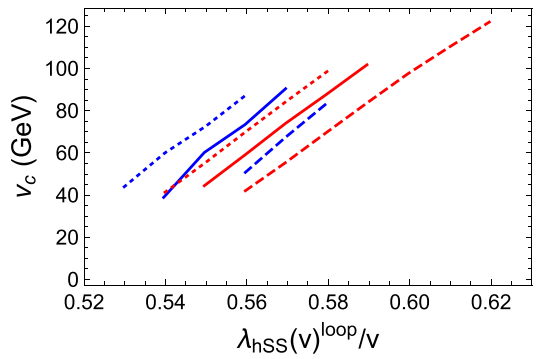

(b) $v_{c}$

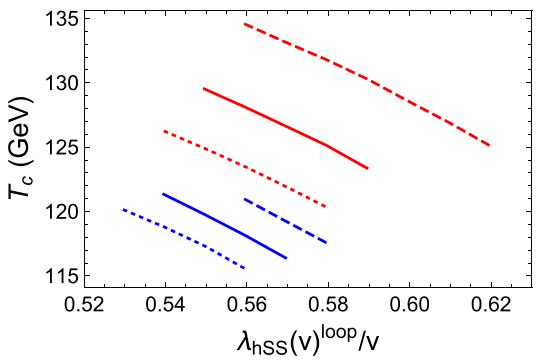

(c) $T_{c}$

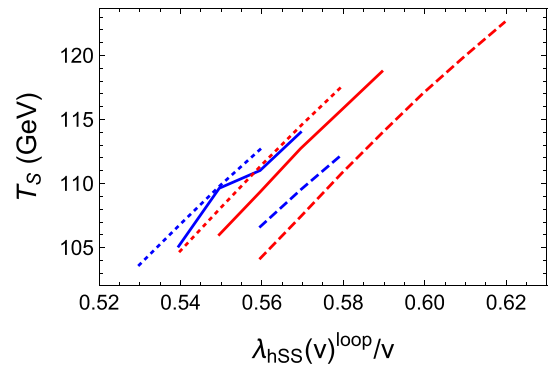

(d) $T_{S}$

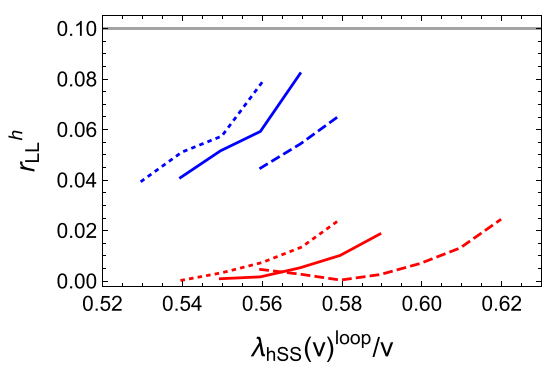

(e) $r_{L L}^{h}$

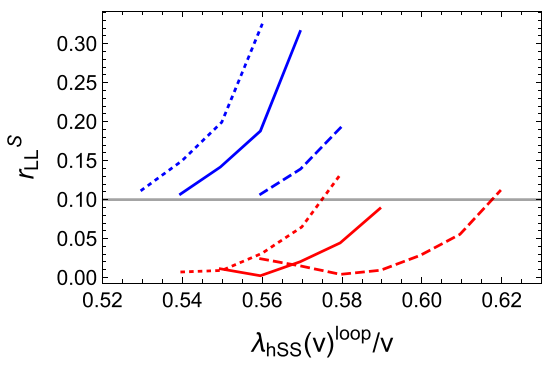

(f) $r_{L L}^{S}$

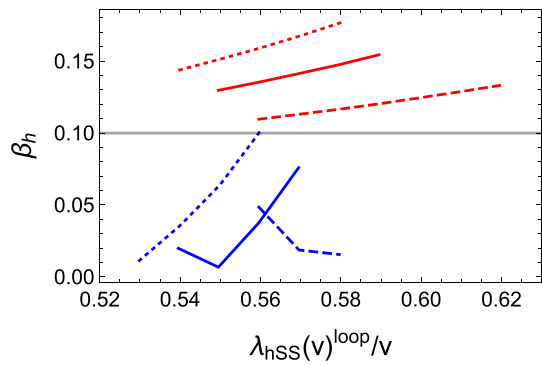

(g) $\beta_{h}$

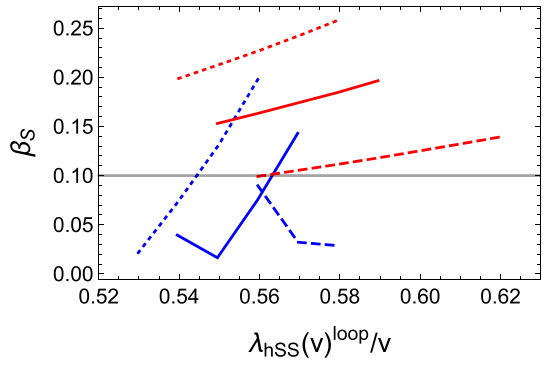

(h) $\beta_{S}$

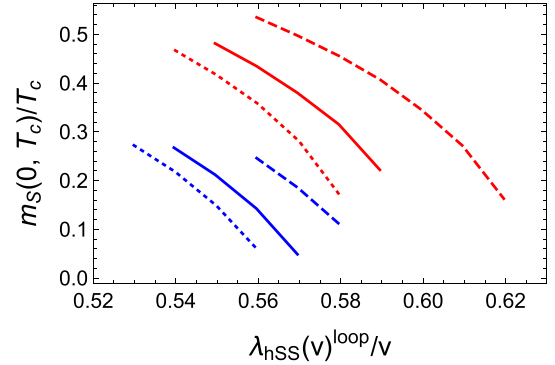

(i) $m_{S}$ at origin

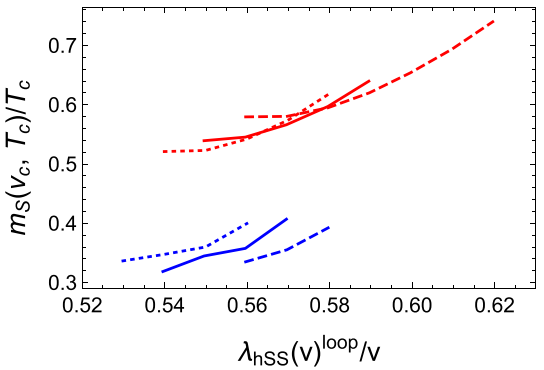

(j) $m_{S}$ at $v_{c}$

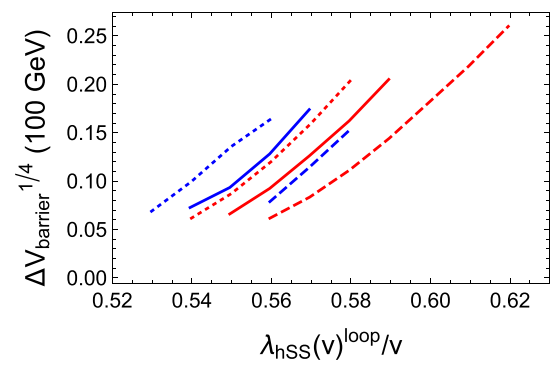

(k) potential barrier

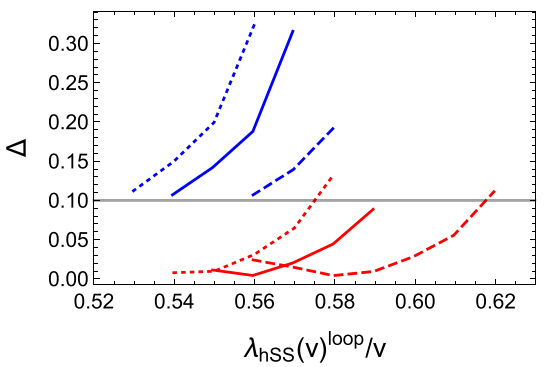

(l) Largest error term $\Delta^{\mathrm{TFD}, \mathrm{PD}}$
Fig. 4 Comparison of one-step phase transition in the new PD (blue) vs the standard TFD (red) calculation, for $N_{S}=6$ and $\left(m_{S}, \lambda_{S}^{\text {loop }}\right)=$ (150 GeV, 1.0). The renormalization scale is set to $\mu_{R}=m_{S}$ (solid lines). Dashed (dotted) lines correspond to $m u_{R}=2 m_{S}\left(m_{S} / 2\right)$ to demonstrate the effect of scale variation. To the left of the curves, the

understood as the solution to the equations $\mu^{2}=\lambda_{H S} T^{2}$ and $-\mu_{S}^{2}=\lambda_{H S} T^{2}$ (neglecting numerical prefactors). This explains why $T_{c}$ decreases with increasing Higgs portal coupling, but is insufficient to understand why $T_{S}$ increases, since
PT is one-step and weakly first order or second order. To the right of the curves, $T_{S}>T_{c}$ and the transition is two-step for $\lambda_{h S S}(v)^{\text {loop }}<\lambda_{h S S}^{\max }$. This upper bound is set by the condition that EWSB vacuum is preferred and depends on $N_{S}, m_{S}, \lambda_{S}^{\text {loop }}, \mu_{R}$ but not the choice of thermal resummation scheme

both $\mu_{S}^{2}$ and $\delta m_{S}^{2}$ depend linearly on $\lambda_{H S}$ at leading order in temperature for fixed physical singlet mass $m_{S}$. Solving for $T_{S}$ with the full high-temperature expansion of the thermal potential reproduces the behavior shown in (d). As the Higgs 
portal coupling is further increased, $T_{S}$ becomes larger than $T_{c}$, which occurs around $\lambda_{h S S}^{\text {loop }} / v \approx 0.6$ in Fig. 4 . This means the phase transition is now two-step: as the universe cools it falls first into the $(h, S)=(0, w)$ vacuum before transitioning to the $(v, 0)$ vacuum. Increasing $\lambda_{H S}$ decreases the potential difference $V_{\text {eff }}^{T=0}(v, 0)-V_{\text {eff }}^{T=0}(0, w)$, which delays the second transition, and by lowering $T_{c}$ enhances $v_{c} / T_{c}$. Two-step phase transitions can therefore be very strong, since they can rely on supercooling the universe. Finally, as $\lambda_{H S}$ is increased further still the $(h, S)=(0, w)$ vacuum becomes preferred to our vacuum at zero temperature, and the model is not compatible with our universe.

With this understanding, we can now interpret the differences between the standard TFD and the new (O)PD calculation in detail:

- (O)PD and TFD predict different parameter regions where the one-step phase transition is strongly first order. This arises due to three effects: in (O)PD, $v_{c}$ is larger, $T_{c}$ is smaller, and $T_{S}$ is larger than in TFD. The first and second effect make the PT stronger at a given parameter point, but the second and third effect lead to a lower Higgs portal coupling at which the switch from one-step to two-step PT occurs.

The third effect can be traced back to the smaller thermal mass corrections obtained in (O)PD, while the first two effects are also connected to the $h$-dependence of the mass correction (see Fig. 3).

As a result, the region of parameter space in which (O)PD predicts a strong first order one-step PT with $v_{c} / T_{c}>0.6$ is much smaller than in TFD, and shifted to smaller Higgs portal couplings. By the same token, the region of parameter space where the PT is two-step (to the right of the curves in Fig. 4) is larger in (O)PD.

- The finite temperature singlet mass at the origin and at $v_{c}$, shown in Fig. 4(i) and (j), shows that the high-temperature approximation is fairly reliable in its untruncated form (since $m / T \lesssim 1$ ), but the truncated high- $T$ approximation assumed in the TFD thermal mass calculation makes errors of $m / T \sim 30$ to $70 \%$ depending on $h$, which is consistent with Fig. 3(right).

- The Higgs portal dependent curves of (b) $v_{c}$ and (k) potential barrier height of the $(\mathrm{O}) \mathrm{PD}$ calculation are very similar to TFD curves that are shifted to lower couplings. The same holds for $v_{c} / T_{c}$, which is controlled by the rapidly-varying $v_{c}$. Therefore, we expect the ratio $T_{n} / T_{c}$ of the nucleation temperature, when the bubbles of true vacuum actually form, to the critical temperature to be similar in the two calculation.

- The absolute value of the critical temperature $T_{c}$ for a given strength of phase transition $v_{c} / T_{c}$ is $\sim 10 \%$ lower than predicted by the TFD calculation. If a one-step transition could be strong enough to be detected by gravita- tional wave observations, this would effect the frequency spectrum of the stochastic gravitational wave signal.

- In the example of Fig. 4, the overall error term (1) $\triangle^{T F D, P D}$ is dominated by the singlet lollipop ratio (f) $r_{L L}^{S}$. The error term is much larger in the new (O)PD calculation than the standard TFD. Since the latter underestimates the error, we expect $\Delta^{\mathrm{PD}}$ to give a much better representation of the calculation's reliability, which is breaking down near the switch from one-step to two-step phase transitions (larger $\lambda_{h S S}^{\text {loop }} / v$ ).

For other slices of parameter space, the $\beta$-errors can dominate, in which case the $(\mathrm{O}) \mathrm{PD}$ calculation can be much more reliable, since the first neglected contributions are $\mathcal{O}\left(\beta^{3}\right)$.

It is now straightforward to interpret Fig. 5, which shows the regions of the $\lambda_{h S S}^{\text {loop }}-\lambda_{S}^{\text {loop }}$ coupling plane that give a strong one- or two-step first order EWPT in the TFD and (O)PD calculations for $N_{S}=3$ and $m_{S}=250 \mathrm{GeV}$. In the left plot, a strong one-step transition is defined with the maximally permissive criterion $v_{c} / T_{c}>0.6$. In the right plot, the criterion is slightly tightened, to the usual $v_{c} / T_{c}>1.0$. In the more correct $(\mathrm{O}) \mathrm{PD}$ calculation, the region allowing for a strong one-step transition (between blue lines) is smaller than in TFD (red lines), while the region with a strong two-step transition (between the blue/red lines and the green line) is larger in (O)PD. This is especially pronounced when $v_{c} / T_{c}$ is required to be larger than 1 , in which case there is almost no overlap between the two region with a strong one-step phase transition.

For the specific $\mathrm{SM}+N_{S} \times S$ benchmark model, the collider phenomenology depends almost exclusively on the Higgs portal coupling and the singlet mass. Figure 6 shows the parameter regions where, for some choices of the Singlet quartic $\lambda_{S}$, the one- or two-step EWPT can be strong enough for EWBG. This generalizes the results of [111] to $N_{S} \geq 1$ and makes clear that future colliders will be able to probe the entire parameter space of this representative class of models for arbitrary number of singlets.

In this particular benchmark model, the singlet quartic and the Higgs portal coupling are free parameters, and the lack of mixing with the Higgs makes the physical effects of the singlet quartic very hard to observe at colliders. Therefore, the old TFD and more correct (O)PD calculations give very similar predictions for the collider phenomenology of EWBG. However, as discussed above, the situation would be very different in more complete theories, especially if a full calculation of the baryon asymmetry reveals that $v_{c} / T_{c}$ has to be larger than 0.6. Furthermore, even in the SM+ $N_{S} \times S$ benchmark model, there is an important difference: the minimum singlet mass required for a strong one-step PT is higher in $(\mathrm{O}) \mathrm{PD}$ than in TFD. This means that for light singlet masses, the only possibility for EWBG is via a two-step 


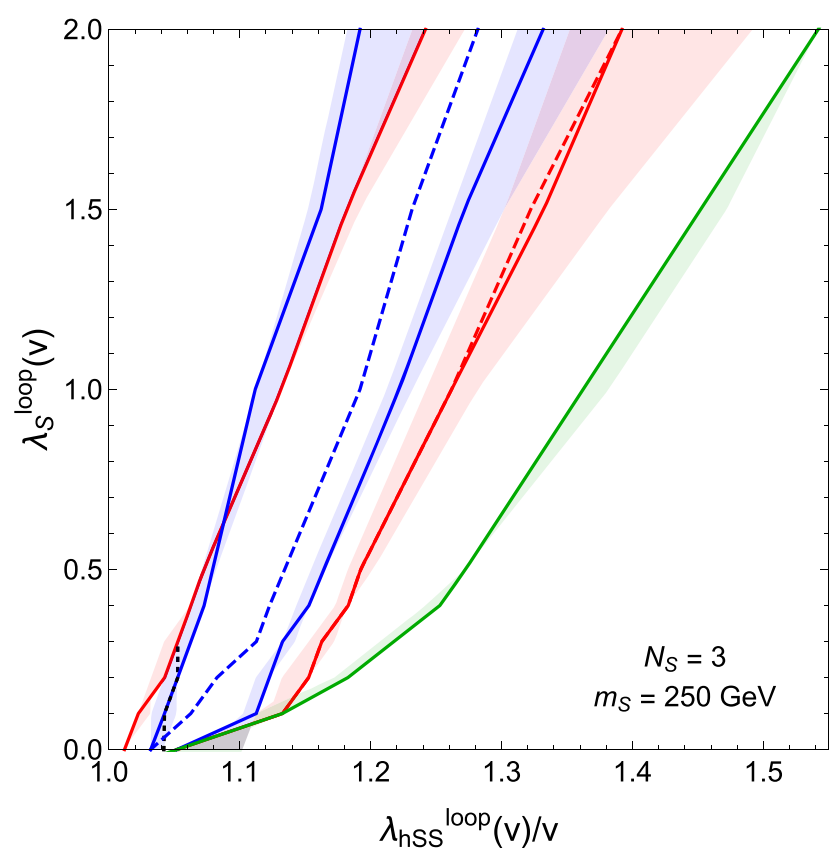

Fig. $5 \mathrm{SM}+N_{S} \times S$ parameter space with a strong EWPT for $N_{S}=3$ and $m_{S}=250 \mathrm{GeV}$. Region between solid red (blue) lines: regions with strong one-step PT satisfying $v_{c} / T_{c}>0.6$ for the standard TFD (new PD) calculation on the left, and $v_{c} / T_{c}>1.0$ on the right. To the left of these lines, the PT is weakly first order or second order. Between the red (blue) lines and the green line, the PT is two-step in TFD (PD) calculation. To the right of the green line, the EWSB vacuum is not

transition. As discussed in [118], these transitions can form runaway bubbles of true vacuum, which do not permit successful baryon number generation, and further study could reveal very strong additional constraints on the parameter space actually compatible with complete EWBG. Furthermore, the stochastic gravitational wave background generated by a strong two-step transition, but not a moderately strong one-step transition, could be detected by gravitational wave observatories [113]. The (O)PD calculation reveals this exciting possibility to be more likely for light singlet masses than previously assumed.

\section{Conclusions}

In this paper we developed the partial dressing and optimized partial dressing schemes for computation and resummation of thermal masses beyond the high-temperature approximation in general BSM scenarios. This allows for the strength of Phase Transitions to be determined to much greater accuracy than the standard Truncated Full Dressing scheme, which only resums hard thermal loops by inserting $\Pi_{i} \sim T^{2}$ into the effective potential.

Our phenomenological analysis of the EWPT in the SM $+N_{S} \times S$ benchmark model generalizes the results of [111],

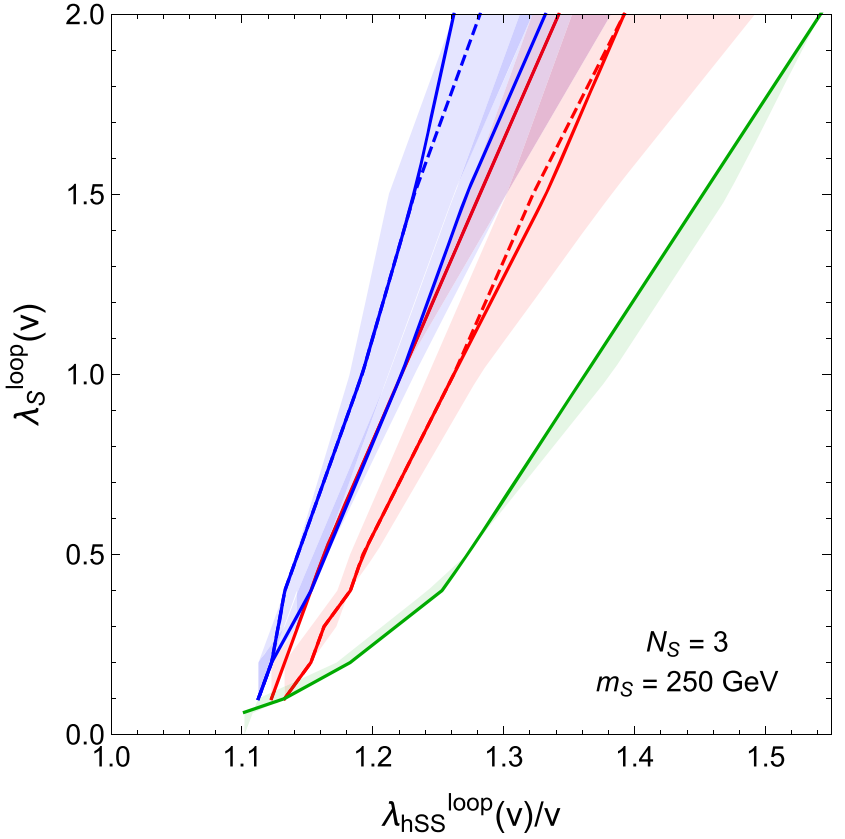

preferred at zero temperature (this does not depend on the thermal mass resummation scheme). Varying $\mu_{R}$ between 0.5 and $2 m_{S}$ gives the variation indicated by the red/blue/green shading. To the right of the red (blue) dashed lines, $\Delta^{\mathrm{TFD}}\left(\Delta^{\mathrm{PD}}\right)>0.1$ for $\mu_{R}=m_{S}$. To the left of the black dotted line, the singlet is stable at the origin at zero temperature for $\mu_{R}=1$

and shows that EWBG in singlet extensions without Higgs mixing is guaranteed to be discovered at future $100 \mathrm{TeV}$ and lepton colliders. Given that more general models with Higgs mixing generate additional signatures which are expected to be detectable at a future $100 \mathrm{TeV}$ collider [117], the outlook for a general phenomenological no-lose theorem is optimistic, though more work is needed to make this conclusion completely robust.

The (O)PD calculation shows two-step phase transitions are more prevalent than previously assumed from TFD calculations. This is encouraging, as strong two-step transitions can generate observable gravity wave signals [113]. They are also more constrained, since runaway bubbles are incompatible with baryon number generation [118]. Further analysis is needed to determine whether this translates to additional constraints on the $\mathrm{SM}+N_{S} \times S$ benchmark model.

The OPD scheme represents a simple extension on the standard TFD calculation, takes only slightly more CPU time to solve for the strength of the phase transition, and is easy to implement in Mathematica. We supply a condensed instruction manual in Appendix A. We hope that the OPD calculation will be useful in the future study of other BSM scenarios. This is particularly motivated, since for more complete theories with additional correlations amongst 

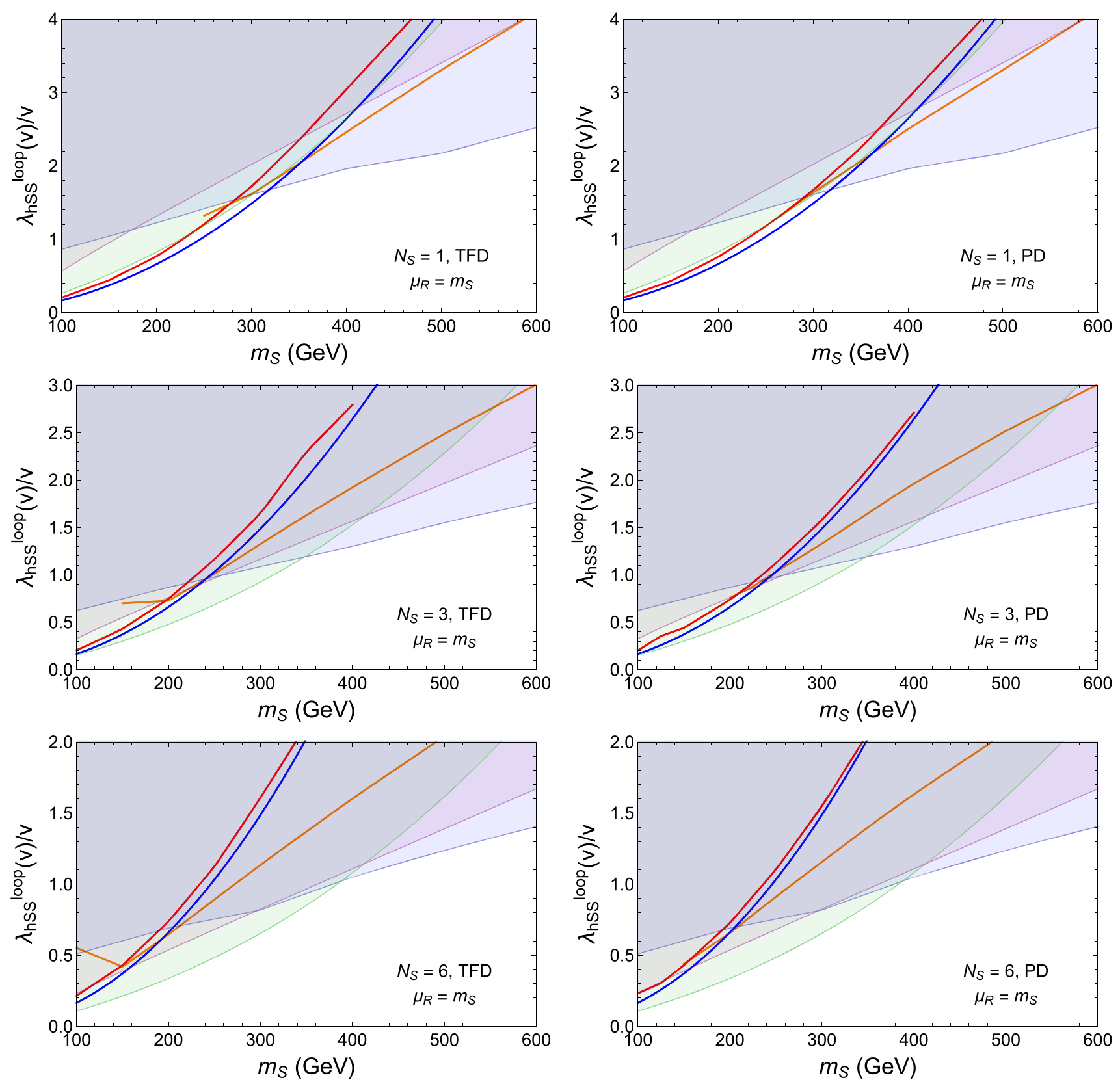

$$
\begin{aligned}
& \delta \lambda_{\text {hhh }}>10 . \% \\
& \mathrm{~S} / \sqrt{\mathrm{B}}>2 @ 100 \mathrm{TeV}, 30 / \mathrm{ab} \\
& \delta \sigma_{\mathrm{Zh}}>0.006
\end{aligned}
$$

Fig. 6 Phenomenological parameter space of the $\mathrm{SM}+N_{S} \times S$ benchmark model of EWBG, for $N_{S}=1,3,6$, computed in the TFD or PD thermal resummation scheme. The physical singlet mass in our EWSB vacuum is on the horizontal axis, the higgs-singlet cubic coupling is on the vertical axis. This determines most collider observables. The Higgs cubic coupling deviation Eq. (4.5) is bigger than $10 \%$ in the blue shaded region, to which a $100 \mathrm{TeV}$ collider with $30 \mathrm{ab}^{-1}$ has more than $2 \sigma$ sensitivity [97]. For the same luminosity, a direct VBF + MET
— below line, Singlet stable at origin

- above line: one-step $\mathrm{v}_{\mathrm{c}} / \mathrm{T}_{\mathrm{c}}>0.6$

— above line: two-step

search for invisible singlet pair production can exclude the green shaded region [111]. In the purple shaded region, a TLEP-like lepton collider can probe the $Z h$ cross section deviation, see Eq. (4.6), at the $2 \sigma$ level. Below the blue line, the singlet is stable at the origin. Above the orange or red solid lines, a one-step or two-step phase transition strong enough for EWBG can occur $\left(\mu_{R}=m_{S}\right)$. In this projected-down parameter space, the effect of scale variation is minor 
the low-energy parameters than in our benchmark model, the OPD calculation makes significantly different predictions for the EWBG-viable parameter space, and hence the associated collider and cosmological observables. In some cases, scenarios which were thought to be viable may now be excluded.

Developing the $(\mathrm{O}) \mathrm{PD}$ thermal resummation scheme is a necessary ingredient for the careful study of EFTs at higher temperature, which in turn would represent a great leap in our model-independent understanding of EWBG. We are currently conducting such an analysis, and will present the results in a future publication.

Acknowledgements We thank Thomas Konstandin, Jonathan Kozaczuk, David Morrissey, Jose Miguel No, Hiren Patel, Michael RamseyMusolf, Carlos Tamarit and the participants of the MIAPP workshop "Why is there more Matter than Antimatter in the Universe?" for useful discussions. The work of DC was supported in part by the Munich Institute for Astro- and Particle Physics (MIAPP) of the DFG cluster of excellence "Origin and Structure of the Universe". D.C. is supported by National Science Foundation Grant No. PHY-1315155 and the Maryland Center for Fundamental Physics. P.M and H.R. are supported in part by NSF CAREER award NSF-PHY-1056833 and NSF award NSFPHY-1620628.

Open Access This article is distributed under the terms of the Creative Commons Attribution 4.0 International License (http://creativecomm ons.org/licenses/by/4.0/), which permits unrestricted use, distribution, and reproduction in any medium, provided you give appropriate credit to the original author(s) and the source, provide a link to the Creative Commons license, and indicate if changes were made.

Funded by SCOAP ${ }^{3}$.

\section{Appendix A: Instruction manual for optimized partial dressing calculation of phase transition}

Here we summarize the detailed procedure for obtaining the effective finite-temperature potential $V_{\mathrm{eff}}^{\mathrm{pd}}(h, T)$ as a function of $h$ for a given temperature $T$ in the optimized partial dressing (OPD) scheme. This will be familiar to anyone studying the EWPT in BSM theories, and implementing the OPD calculation in Mathematica is very similar to the familiar TFD calculation, and only $\sim \mathcal{O}(10 \%)$ more CPU intensive. We explain this procedure in the context of the $\mathrm{SM}+N_{S} \times S$ benchmark model, but it generalizes easily to other theories with one-step phase transitions. At every point, only use the real parts of various potential contributions or their derivatives. Note that we do not perform this resummation for zero temperature matching and other calculations, since the effect is small.

For a given temperature, we first have to find the thermal mass corrections $\delta m_{i}^{2}(h, T)$. For gauge bosons, use the standard $\delta m_{i}^{2}=\Pi_{i} \sim T^{2}$, see e.g. Eq. (2.22) for the SM contributions. For scalars we have to solve a set of coupled gap equations:

$$
\begin{aligned}
\delta m_{\phi_{j}}^{2}(h, T)= & \sum_{i} \frac{\partial}{\partial \phi_{j}}\left[\frac{\partial V_{\mathrm{CW}}^{i}}{\partial \phi_{j}}\left(m_{i}^{2}(h)+\delta m_{i}^{2}(h, T)\right)\right. \\
& \left.+\frac{\partial V_{\mathrm{th}}^{i}}{\partial \phi_{j}}\left(m_{i}^{2}(h)+\delta m_{i}^{2}(h, T), T\right)\right] .
\end{aligned}
$$

See Eqs. (2.6) and (2.11) for definitions of the ColemanWeinberg and finite-temperature potential. For the latter, use the high-temperature expansion to log-order in the gap equation, see Eq. (2.13). Note that this makes the RHS a set of fully analytical expressions that can be easily manipulated in Mathematica.

In our specific benchmark model, there are three gap equations: for $j=h, G_{0}, S_{0}$. Since we are interested in excursions along the $h$-axis, all Goldstone thermal masses are the same but different from the physical Higgs mass, and all scalar thermal masses are the same. ${ }^{14}$ Note that the sum $i$ also runs over all particles, including fermions and gauge bosons.

We numerically solve this set of gap equations by essentially treating it as a set of partial differential equations. Specifically, set up a coarse grid $^{15}$ along the Higgs axis $h=\{0,10,20, \ldots, 250\}$. The RHS of the gap equation is a function of thermal masses and their derivatives. Expanding the mass correction around $h=h_{a}$ :

$\delta m_{j}^{2}(h, T) \approx \delta m_{j(a)}^{2}+\left(h-h_{a}\right) \frac{\partial \delta m_{j(a)}^{2}}{\partial h}$

and substituting this form of $\delta m_{h, G_{0}, S}^{2}$ (as well as the $h$ independent gauge boson thermal masses) into the RHS yields three gap equations that depend on six parameters: the value of the scalar thermal masses $\delta m_{h(a)}^{2}, \delta m_{G_{0}(a)}^{2}, \delta m_{S(a)}^{2}$ and their first derivatives $\partial \delta m_{h(a)}^{2} / \partial h, \partial \delta m_{G_{0}(a)}^{2} / \partial h, \partial \delta$ $m_{S(a)}^{2} / \partial h$.

At the origin, the derivatives are zero, and solving for just the three thermal masses yields a numerically unique set of solutions. Then we work our way away from the origin. The solution at $h=h_{a}$ can be obtained by first eliminating either the derivatives or the actual thermal masses from the gap equation using the continuity condition

$\delta m_{j(a-1)}^{2} \approx \delta m_{j(a)}^{2}+\left(h_{a-1}-h_{a}\right) \frac{\partial \delta m_{j(a)}^{2}}{\partial h}$

given the known solution at $h=h_{a-1}$. We found in practice that eliminating the $\delta m_{j(a)}^{2}$ and solving for the three derivatives $\partial \delta m_{j(a)}^{2} / \partial h$ was more reliable. If the solution fails the

\footnotetext{
${ }^{14}$ If we solved for excursions along the $S_{0}$ direction, we would have to treat $S_{0}$ and $S_{k>0}$ differently but could treat all Higgs degrees of freedom the same.

15 To avoid singularities the origin can be defined to be a very small positive displacement instead of identically zero.
} 
resolution of the grid may have to be increased, but we found a coarse grid with $10 \mathrm{GeV}$ spacing to be sufficient. ${ }^{16}$

Once a grid of solutions is obtained, the continuous functions $\delta m_{j}^{2}(h, T)$ are defined by linear interpolation. The thermal potential is then defined by

$$
\begin{aligned}
V_{\mathrm{eff}}^{\mathrm{pd}}(h, T)= & V_{0}+\sum_{i} \int d h\left[\frac{\partial V_{\mathrm{CW}}^{i}}{\partial h}\left(m_{i}^{2}(h)+\delta m_{i}^{2}(h, T)\right)\right. \\
& \left.+\frac{\partial V_{\mathrm{th}}^{i}}{\partial h}\left(m_{i}^{2}(h)+\delta m_{i}^{2}(h, T), T\right)\right], \quad \text { (A.4) }
\end{aligned}
$$

where $i$ runs over all scalars, gauge bosons and fermions (with zero thermal mass for the latter). Importantly, in this effective potential, use the piece-wise defined thermal functions

$$
\begin{aligned}
& J_{B}^{\text {piece-wise }}\left(y^{2}\right)= \begin{cases}J_{B}^{\mathrm{high}-T}\left(y^{2}\right) & \text { for } y^{2} \leq 1.22 \\
\tilde{J}_{B}^{(3)}\left(y^{2}\right) & \text { for } y^{2}>1.22\end{cases} \\
& J_{F}^{\text {piece-wise }}\left(y^{2}\right)= \begin{cases}J_{F}^{\mathrm{high}-T}\left(y^{2}\right) & \text { for } y^{2} \leq 1.29 \\
\tilde{J}_{F}^{(2)}\left(y^{2}\right) & \text { for } y^{2}>1.29\end{cases}
\end{aligned}
$$

See Eqs. (2.13) and (2.14) for the definition of the approximate thermal functions. The integrand can then be defined as an analytical function in Mathematica, which can be evaluated on a grid of Higgs values $h=\{0,5,10, \ldots, 250 \mathrm{GeV}\}$ and interpolated to efficiently perform the integral at arbitrary values of $h<250 \mathrm{GeV}$.

\section{References}

1. Y. Akiba et al., The hot QCD white paper: exploring the phases of QCD at RHIC and the LHC. arXiv:1502.0273

2. Planck Collaboration, P.A.R. Ade et al., Planck 2015 results. XIII. Cosmological parameters. Astron. Astrophys. 594, A13 (2016). arXiv: 1502.0158

3. E.W. Kolb, M.S. Turner, The early universe. Front. Phys. 69, 1547 (1990)

4. A.D. Sakharov, Violation of CP invariance, c asymmetry, and baryon asymmetry of the universe. Pisma Zh. Eksp. Teor. Fiz. 5, 32-35 (1967)

5. A.D. Sakharov, Violation of $\mathrm{CP}$ invariance, c asymmetry, and baryon asymmetry of the universe. Usp. Fiz. Nauk. 161, 61 (1991)

6. V. Kuzmin, V. Rubakov, M. Shaposhnikov, On the anomalous electroweak baryon number nonconservation in the early universe. Phys. Lett. B 155, 36 (1985)

7. F.R. Klinkhamer, N. Manton, A saddle point solution in the Weinberg-Salam theory. Phys. Rev. D 30, 2212 (1984)

8. M.E. Shaposhnikov, Possible appearance of the baryon asymmetry of the universe in an electroweak theory. JETP Lett. 44, 465468 (1986)

9. M.E. Shaposhnikov, Possible appearance of the baryon asymmetry of the universe in an electroweak theory. Pisma Zh. Eksp. Teor. Fiz. 44, 364 (1986)

\footnotetext{
16 The automatic Mathematica functions for differential equation solving failed with these gap equations, possibly by over-interpreting unphysical local singularities or numerical noise. We found our manual implementation to be very simple, fast and reliable.
}

10. M.E. Shaposhnikov, Baryon asymmetry of the universe in standard electroweak theory. Nucl. Phys. B 287, 757-775 (1987)

11. P.B. Arnold, L.D. McLerran, Sphalerons, small fluctuations and baryon number violation in electroweak theory. Phys. Rev. D 36, 581 (1987)

12. P.B. Arnold, L.D. McLerran, The sphaleron strikes back. Phys. Rev. D 37, 1020 (1988)

13. S.Y. Khlebnikov, M. Shaposhnikov, The statistical theory of anomalous fermion number nonconservation. Nucl. Phys. B 308, 885-912 (1988)

14. J.M. Cline, Baryogenesis. arXiv:hep-ph/0609145

15. M. Trodden, Electroweak baryogenesis. Rev. Mod. Phys. 71, 1463-1500 (1999). arXiv:hep-ph/9803479

16. A. Riotto, Theories of baryogenesis. arXiv:hep-ph/9807454

17. A. Riotto, M. Trodden, Recent progress in baryogenesis. Annu. Rev. Nucl. Part. Sci. 49, 35-75 (1999). arXiv:hep-ph/9901362

18. M. Quiros, Finite temperature field theory and phase transitions. arXiv:hep-ph/9901312

19. D.E. Morrissey, M.J. Ramsey-Musolf, Electroweak baryogenesis. New J. Phys. 14, 125003 (2012). arXiv: 1206.2942

20. L. Dolan, R. Jackiw, Symmetry behavior at finite temperature. Phys. Rev. D 9, 3320-3341 (1974)

21. S. Weinberg, Gauge and global symmetries at high temperature. Phys. Rev. D 9, 3357-3378 (1974)

22. A.I. Bochkarev, M.E. Shaposhnikov, Electroweak production of baryon asymmetry and upper bounds on the higgs and top masses. Mod. Phys. Lett. A 2, 417 (1987)

23. K. Kajantie, M. Laine, K. Rummukainen, M.E. Shaposhnikov, The electroweak phase transition: a nonperturbative analysis. Nucl. Phys. B 466, 189-258 (1996). arXiv:hep-lat/9510020

24. S. Profumo, M.J. Ramsey-Musolf, G. Shaughnessy, Singlet Higgs phenomenology and the electroweak phase transition. JHEP 0708 , 010 (2007). arXiv:0705.2425

25. A. Ashoorioon, T. Konstandin, Strong electroweak phase transitions without collider traces. JHEP 0907, 086 (2009). arXiv:0904.0353

26. P.H. Damgaard, D. O'Connell, T.C. Petersen, A. Tranberg, Constraints on new physics from baryogenesis and large hadron collider data. Phys. Rev. Lett. 111(22), 221804 (2013). arXiv: 1305.4362

27. V. Barger, P. Langacker, M. McCaskey, M.J. Ramsey-Musolf, G. Shaughnessy, LHC phenomenology of an extended standard model with a real scalar singlet. Phys. Rev. D 77, 035005 (2008). arXiv:0706.4311

28. J.R. Espinosa, T. Konstandin, F. Riva, Strong electroweak phase transitions in the standard model with a singlet. Nucl. Phys. B 854, 592-630 (2012). arXiv:1107.5441

29. A. Noble, M. Perelstein, Higgs self-coupling as a probe of electroweak phase transition. Phys. Rev. D 78, 063518 (2008). arXiv:0711.3018

30. J.M. Cline, K. Kainulainen, Electroweak baryogenesis and dark matter from a singlet Higgs. JCAP 1301, 012 (2013). arXiv: 1210.4196

31. J.M. Cline, K. Kainulainen, P. Scott, C. Weniger, Update on scalar singlet dark matter. Phys. Rev. D 88, 055025 (2013). arXiv: 1306.4710

32. T. Alanne, K. Tuominen, V. Vaskonen, Strong phase transition, dark matter and vacuum stability from simple hidden sectors. arXiv: 1407.0688

33. J.R. Espinosa, M. Quiros, Novel effects in electroweak breaking from a hidden sector. Phys. Rev. D 76, 076004 (2007). arXiv:hep-ph/0701145

34. S. Profumo, M.J. Ramsey-Musolf, C.L. Wainwright, P. Winslow, Singlet-catalyzed electroweak phase transitions and precision higgs studies. arXiv: 1407.5342 
35. K. Fuyuto, E. Senaha, Improved sphaleron decoupling condition and the Higgs coupling constants in the real singlet-extended SM. Phys. Rev. D 90, 015015 (2014). arXiv: 1406.0433

36. M. Fairbairn, R. Hogan, Singlet fermionic dark matter and the electroweak phase transition. JHEP 1309, 022 (2013). arXiv: 1305.3452

37. M. Jiang, L. Bian, W. Huang, J. Shu, Impact of a complex singlet: electroweak baryogenesis and dark matter. Phys. Rev. D 93(6), 065032 (2016)

38. M. Pietroni, The electroweak phase transition in a nonminimal supersymmetric model. Nucl. Phys. B 402, 27-45 (1993). arXiv:hep-ph/9207227

39. A. Davies, C. Froggatt, R. Moorhouse, Electroweak baryogenesis in the next-to-minimal supersymmetric model. Phys. Lett. B 372, 88-94 (1996). arXiv:hep-ph/9603388

40. S.J. Huber, T. Konstandin, T. Prokopec, M.G. Schmidt, Electroweak phase transition and baryogenesis in the nMSSM. Nucl. Phys. B 757, 172-196 (2006). arXiv:hep-ph/0606298

41. A. Menon, D. Morrissey, C. Wagner, Electroweak baryogenesis and dark matter in the nMSSM. Phys. Rev. D 70, 035005 (2004). arXiv:hep-ph/0404184

42. S.J. Huber, T. Konstandin, T. Prokopec, M.G. Schmidt, Baryogenesis in the MSSM, nMSSM and NMSSM. Nucl. Phys. A 785, 206-209 (2007). arXiv:hep-ph/0608017

43. W. Huang, Z. Kang, J. Shu, P. Wu, J. M. Yang, New insights of electroweak phase transition in NMSSM. arXiv:1405.1152

44. J. Kozaczuk, S. Profumo, L.S. Haskins, C.L. Wainwright, Cosmological phase transitions and their properties in the NMSSM. arXiv: 1407.4134

45. G.C. Dorsch, S.J. Huber, J.M. No, A strong electroweak phase transition in the 2HDM after LHC8. JHEP 10, 029 (2013). arXiv: 1305.6610

46. G.C. Dorsch, S.J. Huber, K. Mimasu, J.M. No, Echoes of the electroweak phase transition: discovering a second Higgs doublet through $A_{0} \rightarrow Z H_{0}$. Phys. Rev. Lett 113(21), 211802 (2014). arXiv: 1405.5537

47. H.H. Patel, M.J. Ramsey-Musolf, Stepping into electroweak symmetry breaking: phase transitions and Higgs phenomenology. Phys. Rev. D 88, 035013 (2013). arXiv: 1212.5652

48. N. Blinov, J. Kozaczuk, D.E. Morrissey, C. Tamarit, Electroweak baryogenesis from exotic electroweak symmetry breaking. Phys. Rev. D 92(3), 035012 (2015). arXiv:1504.0519

49. S. Inoue, G. Ovanesyan, M.J. Ramsey-Musolf, Two-step electroweak baryogenesis. Phys. Rev. D 93, 015013 (2016). arXiv: 1508.0540

50. M.S. Carena, M. Quiros, C. Wagner, Opening the window for electroweak baryogenesis. Phys. Lett. B 380, 81-91 (1996). arXiv:hep-ph/9603420

51. M. Laine, K. Rummukainen, The MSSM electroweak phase transition on the lattice. Nucl. Phys. B 535, $423-457$ (1998). arXiv:hep-lat/9804019

52. J. Espinosa, Dominant two loop corrections to the MSSM finite temperature effective potential. Nucl. Phys. B 475, 273-292 (1996). arXiv:hep-ph/9604320

53. D. Delepine, J. Gerard, R. Gonzalez Felipe, J. Weyers, A light stop and electroweak baryogenesis. Phys. Lett. B 386, 183-188 (1996). arXiv:hep-ph/9604440

54. M.S. Carena, M. Quiros, C. Wagner, Electroweak baryogenesis and Higgs and stop searches at LEP and the Tevatron. Nucl. Phys. B 524, 3-22 (1998). arXiv:hep-ph/9710401

55. S. Huber, P. John, M. Schmidt, Bubble walls, CP violation and electroweak baryogenesis in the MSSM. Eur. Phys. J. C 20, 695711 (2001). arXiv:hep-ph/0101249

56. M.S. Carena, M. Quiros, M. Seco, C. Wagner, Improved results in supersymmetric electroweak baryogenesis. Nucl. Phys. B 650, 24-42 (2003). arXiv:hep-ph/0208043
57. C. Lee, V. Cirigliano, M.J. Ramsey-Musolf, Resonant relaxation in electroweak baryogenesis. Phys. Rev. D 71, 075010 (2005). arXiv:hep-ph/0412354

58. M. Carena, G. Nardini, M. Quiros, C.E. Wagner, The effective theory of the light stop scenario. JHEP 0810, 062 (2008). arXiv:0806.4297

59. M. Carena, G. Nardini, M. Quiros, C. Wagner, The baryogenesis window in the MSSM. Nucl. Phys. B 812, 243-263 (2009). arXiv:0809.3760

60. V. Cirigliano, Y. Li, S. Profumo, M.J. Ramsey-Musolf, MSSM baryogenesis and electric dipole moments: an update on the phenomenology. JHEP 1001, 002 (2010). arXiv:0910.4589

61. D. Curtin, P. Jaiswal, P. Meade, Excluding electroweak baryogenesis in the MSSM. JHEP 1208, 005 (2012). arXiv:1203.2932

62. T. Cohen, D.E. Morrissey, A. Pierce, Electroweak baryogenesis and Higgs signatures. Phys. Rev. D 86, 013009 (2012). arXiv: 1203.2924

63. A. Katz, M. Perelstein, Higgs couplings and electroweak phase transition. arXiv: 1401.1827

64. A. Katz, M. Perelstein, M.J. Ramsey-Musolf, P. Winslow, Stopcatalyzed baryogenesis beyond the MSSM. Phys. Rev D92(9), 095019 (2015). arXiv: 1509.0293

65. J.M. Cline, K. Kainulainen, A new source for electroweak baryogenesis in the MSSM. Phys. Rev. Lett. 85, 5519-5522 (2000). arXiv:hep-ph/0002272

66. G.D. Moore, Electroweak bubble wall friction: analytic results JHEP 0003, 006 (2000). arXiv:hep-ph/0001274

67. P. John, M. Schmidt, Do stops slow down electroweak bubble walls? Nucl. Phys. B 598, 291-305 (2001). arXiv:hep-ph/0002050

68. P. John, M. Schmidt, Bubble wall velocity in the MSSM. arXiv:hep-ph/0012077

69. A. Megevand, A.D. Sanchez, Velocity of electroweak bubble walls. Nucl. Phys. B 825, 151-176 (2010). arXiv:0908.3663

70. J. Moreno, M. Quiros, M. Seco, Bubbles in the supersymmetric standard model. Nucl. Phys. B 526, 489-500 (1998). arXiv:hep-ph/9801272

71. A. Riotto, The more relaxed supersymmetric electroweak baryogenesis. Phys. Rev. D 58, 095009 (1998). arXiv:hep-ph/9803357

72. V. Cirigliano, M.J. Ramsey-Musolf, S. Tulin, C. Lee, Yukawa and tri-scalar processes in electroweak baryogenesis. Phys. Rev. D 73, 115009 (2006). arXiv:hep-ph/0603058

73. D.J. Chung, B. Garbrecht, M.J. Ramsey-Musolf, S. Tulin, Yukawa interactions and supersymmetric electroweak baryogenesis. Phys. Rev. Lett. 102, 061301 (2009). arXiv:0808.1144

74. Y. Li, S. Profumo, M. Ramsey-Musolf, Bino-driven electroweak baryogenesis with highly suppressed electric dipole moments. Phys. Lett. B 673, 95-100 (2009). arXiv:0811.1987

75. J.M. Cline, M. Joyce, K. Kainulainen, Supersymmetric electroweak baryogenesis in the WKB approximation. Phys. Lett. B 417, 79-86 (1998). arXiv:hep-ph/9708393

76. J.M. Cline, M. Joyce, K. Kainulainen, Supersymmetric electroweak baryogenesis. JHEP 0007, 018 (2000). arXiv:hep-ph/0006119

77. T. Konstandin, T. Prokopec, M.G. Schmidt, Kinetic description of fermion flavor mixing and CP-violating sources for baryogenesis. Nucl. Phys. B 716, 373-400 (2005). arXiv:hep-ph/0410135

78. T. Konstandin, T. Prokopec, M.G. Schmidt, Axial currents from CKM matrix CP violation and electroweak baryogenesis. Nucl. Phys. B 679, 246-260 (2004). arXiv:hep-ph/0309291

79. J. Kozaczuk, S. Profumo, M.J. Ramsey-Musolf, C.L. Wainwright, Supersymmetric electroweak baryogenesis via resonant sfermion sources. Phys. Rev. D 86, 096001 (2012). arXiv:1206.4100

80. H.H. Patel, M.J. Ramsey-Musolf, Baryon washout, electroweak phase transition, and perturbation theory. JHEP 1107, 029 (2011). arXiv: 1101.4665 
81. D.J. Gross, R.D. Pisarski, L.G. Yaffe, QCD and instantons at finite temperature. Rev. Mod. Phys. 53, 43 (1981)

82. R.R. Parwani, Resummation in a hot scalar field theory. Phys. Rev. D 45, 4695 (1992). arXiv:hep-ph/9204216. [Erratum: Phys. Rev. D 48, 5965 (1993)]

83. P.B. Arnold, O. Espinosa, The effective potential and first order phase transitions: beyond leading-order. Phys. Rev. D 47, 3546 (1993). arXiv:hep-ph/9212235. [Erratum: Phys. Rev. D 50, 6662 (1994)

84. J.R. Espinosa, M. Quiros, F. Zwirner, On the phase transition in the scalar theory. Phys. Lett. B 291, 115-124 (1992). arXiv:hep-ph/9206227

85. J.R. Espinosa, M. Quiros, F. Zwirner, On the nature of the electroweak phase transition. Phys. Lett. B 314, 206-216 (1993). arXiv:hep-ph/9212248

86. M. Quiros, On daisy and superdaisy resummation of the effective potential at finite temperature, in Elementary particle physics. Proceedings, 4th Hellenic School, Corfu, September 2-20, vols. 1\&2, pp. 502-511 (1992). arXiv:hep-ph/9304284

87. C.G. Boyd, D.E. Brahm, S.D.H. Hsu, Resummation methods at finite temperature: the Tadpole way. Phys. Rev. D 48, 4963-4973 (1993). arXiv:hep-ph/9304254

88. M. Dine, R.G. Leigh, P.Y. Huet, A.D. Linde, D.A. Linde, Towards the theory of the electroweak phase transition. Phys. Rev. D 46, 550-571 (1992). arXiv:hep-ph/9203203

89. C.G. Boyd, D.E. Brahm, S.D.H. Hsu, Corrections to the electroweak effective action at finite temperature. Phys. Rev. D 48, 4952-4962 (1993). arXiv:hep-ph/9206235

90. C. Grojean, G. Servant, J.D. Wells, First-order electroweak phase transition in the standard model with a low cutoff. Phys. Rev. D 71, 036001 (2005). arXiv:hep-ph/0407019

91. D. Bodeker, L. Fromme, S.J. Huber, M. Seniuch, The baryon asymmetry in the standard model with a low cut-off. JHEP 02, 026 (2005). arXiv:hep-ph/0412366

92. C. Delaunay, C. Grojean, J.D. Wells, Dynamics of nonrenormalizable electroweak symmetry breaking. JHEP 0804, 029 (2008). arXiv:0711.2511

93. B. Grinstein, M. Trott, Electroweak baryogenesis with a pseudo-goldstone Higgs. Phys. Rev. D 78, 075022 (2008). arXiv:0806.1971

94. D. Asner, T. Barklow, C. Calancha, K. Fujii, N. Graf, et al., ILC Higgs white paper. arXiv:1310.0763

95. ILD Collaboration, J. Tian, K. Fujii, Measurement of Higgs couplings and self-coupling at the ILC. PoS EPS-HEP2013, 316 (2013). arXiv:1311.6528

96. J. Tang et al., Concept for a future super proton-proton collider. arXiv: 1507.0322

97. R. Contino et al., Physics at a $100 \mathrm{TeV}$ pp collider: Higgs and EW symmetry breaking studies. arXiv: 1606.0940

98. A.J. Barr, M.J. Dolan, C. Englert, D.E. Ferreira de Lima, M. Spannowsky, Higgs self-coupling measurements at a $100 \mathrm{TeV}$ hadron collider. JHEP 02, 016 (2015). arXiv: 1412.7154

99. ATLAS-PHYS-PUB-2013-001 (2013). https://cds.cern.ch/ record/1507263

100. J. Baglio, A. Djouadi, R. Gröber, M. Mühlleitner, J. Quevillon, The measurement of the Higgs self-coupling at the LHC: theoretical status. JHEP 1304, 151 (2013). arXiv:1212.5581

101. F. Goertz, A. Papaefstathiou, L.L. Yang, J. Zurita, Higgs Boson self-coupling measurements using ratios of cross sections. JHEP 1306, 016 (2013). arXiv: 1301.3492

102. V. Barger, L.L. Everett, C. Jackson, G. Shaughnessy, Higgspair production and measurement of the triscalar coupling at LHC(8,14). Phys. Lett. B 728, 433-436 (2014). arXiv:1311.2931

103. W. Yao, Studies of measuring Higgs self-coupling with $H H \rightarrow$ $b \bar{b} \gamma \gamma$ at the future hadron colliders. arXiv:1308.6302
104. M.J. Dolan, C. Englert, M. Spannowsky, Higgs self-coupling measurements at the LHC. JHEP 10, 112 (2012). arXiv:1206.5001

105. P. Huang, A. Joglekar, B. Li, C.E.M. Wagner, Probing the electroweak phase transition at the LHC. Phys. Rev D93(5), 055049 (2016). arXiv:1512.0006

106. P.H. Ginsparg, First order and second order phase transitions in gauge theories at finite temperature. Nucl. Phys. B 170, 388-408 (1980)

107. K. Kajantie, K. Rummukainen, M.E. Shaposhnikov, A lattice Monte Carlo study of the hot electroweak phase transition. Nucl. Phys. B 407, 356-372 (1993). arXiv:hep-ph/9305345

108. K. Farakos, K. Kajantie, K. Rummukainen, M.E. Shaposhnikov, 3-d physics and the electroweak phase transition: a framework for lattice Monte Carlo analysis. Nucl. Phys. B 442, 317-363 (1995). arXiv:hep-lat/9412091

109. K. Kajantie, M. Laine, K. Rummukainen, M.E. Shaposhnikov, Generic rules for high temperature dimensional reduction and their application to the standard model. Nucl. Phys. B 458, 90-136 (1996). arXiv:hep-ph/9508379

110. K. Kajantie, M. Laine, K. Rummukainen, M.E. Shaposhnikov, High temperature dimensional reduction and parity violation. Phys. Lett. B 423, 137-144 (1998). arXiv:hep-ph/9710538

111. D. Curtin, P. Meade, C.-T. Yu, Testing electroweak baryogenesis with future colliders. JHEP 11, 127 (2014). arXiv:1409.0005

112. M. Kamionkowski, A. Kosowsky, M.S. Turner, Gravitational radiation from first order phase transitions. Phys. Rev. D 49, 2837 2851 (1994). arXiv:astro-ph/9310044

113. C. Grojean, G. Servant, Gravitational waves from phase transitions at the electroweak scale and beyond. Phys. Rev. D 75, 043507 (2007). arXiv:hep-ph/0607107

114. S.R. Coleman, E.J. Weinberg, Radiative corrections as the origin of spontaneous symmetry breaking. Phys. Rev. D 7, 1888-1910 (1973)

115. D.J. Chung, A.J. Long, L.-T. Wang, The $125 \mathrm{GeV}$ Higgs and electroweak phase transition model classes. Phys. Rev. D 87, 023509 (2013). arXiv:1209.1819

116. M.L. Bellac, Thermal Field Theory (Cambridge University Press, Cambridge, 2011)

117. A.V. Kotwal, M.J. Ramsey-Musolf, J.M. No, P. Winslow, Singletcatalyzed electroweak phase transitions in the $100 \mathrm{TeV}$ frontier. Phys. Rev D94(3), 035022 (2016). arXiv: 1605.0612

118. J. Kozaczuk, Bubble expansion and the viability of singletdriven electroweak baryogenesis. JHEP 10, 135 (2015). arXiv: 1506.0474

119. P.H. Damgaard, A. Haarr, D. O'Connell, A. Tranberg, Effective field theory and electroweak baryogenesis in the singlet-extended standard model. JHEP 02, 107 (2016). arXiv:1512.0196

120. S.P. Martin, Taming the Goldstone contributions to the effective potential. arXiv:1406.2355

121. J. Elias-Miro, J.R. Espinosa, T. Konstandin, Taming infrared divergences in the effective potential. JHEP 08, 034 (2014). arXiv: 1406.2652

122. M.E. Carrington, The effective potential at finite temperature in the Standard Model. Phys. Rev. D 45, 2933-2944 (1992)

123. J. Alwall, R. Frederix, S. Frixione, V. Hirschi, F. Maltoni, O. Mattelaer, H.S. Shao, T. Stelzer, P. Torrielli, M. Zaro, The automated computation of tree-level and next-to-leading order differential cross sections, and their matching to parton shower simulations. JHEP 07, 079 (2014). arXiv: 1405.0301

124. C. Englert, M. McCullough, Modified Higgs sectors and NLO associated production. JHEP 1307, 168 (2013). arXiv:1303.1526

125. N. Craig, C. Englert, M. McCullough, New probe of naturalness. Phys. Rev. Lett 111(12), 121803 (2013) 